\title{
Phenolic Profile and In Vitro Assessment of Cytotoxicity and Antibacterial Activity of Ziziphus spina-christi Leaf Extracts
}

\section{Mohamed Gamaleldin Elsadig Karar, Laura Quiet, Ahmed Rezk, Rakesh Jaiswal, Maren Rehders, Matthias S Ullrich, Klaudia Brix and Nikolai Kuhnert ${ }^{*}$}

Department of Life Science and Chemistry, Jacobs University Bremen, Campus Ring 8, 28759 Bremen, Germany

\begin{abstract}
Background: Ziziphus spina-christi is a tropical evergreen tree of Sudanese origin. The plant is commonly used in ethno-medicine for the treatment of many diseases such as malaria, digestive disorders, weakness, hepatic diseases, obesity, urinary problems, diabetes, skin infections, fever, diarrhea and insomnia. However, the pharmaceutical properties and bioactive compounds present in Z. spina-christi are still unknown. Therefore, the aim of the study was to significantly improve and deepen our knowledge about the phenolic composition of $Z$. spina-christi leaf extracts as well as their antibacterial and potential cytotoxic effects.
\end{abstract}

Methods: The phytochemical profile of the known compounds in the methanol/water $(7: 3 \mathrm{v} / \mathrm{v})$ extract of $Z$. spinachristi leaves were tentatively assigned on the basis of reported analytical data from three to four pieces of independent methods including retention time $\left(t_{\mathrm{p}}\right)$, UV/Vis spectroscopy, high resolution mass spectrometry (HR-MS) and tandem mass spectrometry $\left(\mathrm{MS}^{n}\right)$. Four bioactive compounds were purified via preparative-HPLC and identified by means of HPLC-ESI-TOF, HPLC-ESI-MS ${ }^{n},{ }^{1} \mathrm{H}-\mathrm{NMR},{ }^{13} \mathrm{C}-\mathrm{NMR}$, literature data and authentic reference standards. Antibacterial activities of aqueous and methanol leaf extracts were carried out with six bacterial strains (Bacillus subtilis, Bacillus aquimaris, Clavibacter michiganensis, Escherichia coli, Erwinia amylovora, and Pseudomonas syringae) using an agar diffusion assay. Cytotoxic effects towards the human epidermal keratinocyte cell line $\mathrm{HaCaT}$ and rat intestine epithelial IEC-6 cells was assess by cytoskeletal and plasma membrane integrity or analyses of mitochondrial and proliferative activities.

Results: We tentatively assigned fifty-seven phenolic compounds (fifty-two of them are known) to their regioisomeric level in the methanol/water extract of $Z$. spina-christi leaves. To our knowledge 45 of them were not reported previously in Z. spina-christi and five for the first time in nature. Highly glycosylated flavonoids, proanthocyanidins, and chlorogenic acids were identified as the major components. Preparative-HPLC let to the isolation of four known phytochemicals including quercetin 3-O-(6-O-rhamnosyl-glucoside) (rutin) 26, quercetin 3-O-(2-O-rhamnosyl-arabinoside) 27, phloretin $3^{\prime}, 5^{\prime}$ di-C-glucoside 39 and quercetin 3-O-(2,6-di-O-rhamnosyl-glucoside) 50 . Regarding the antimicrobial assays, the methanol extracts of the plant were found to show higher activity than the aqueous extracts indicating hydrophobic substances of Ziziphus leaves exerting antibacterial activity. The extracts revealed no cytotoxic effects towards the HaCaT or IEC-6 cells.

Conclusions: Several previously known and unknown compounds were indentified in Z. spina-christi leaf extracts. The plant possesses strong antimicrobial activity and no toxicity. Our results therefore suggest that $Z$. spina-christi extracts might be potentially suitable for topical applications and support the use of the plant in traditional medicine to treat infectious diseases.

Keywords: Ziziphus spina-christi; Rhamnaceae; Polyphenols; Antibacterial activity; Cytotoxicity; HPLC-DAD-ESI-MS ${ }^{n}$

\section{Background}

Ziziphus spina-christi (L.) Desf. (Rhamnaceae) is a tropical evergreen tree of Sudanese origin. The plant has very interesting historical and religious aspects. It is repeatedly mentioned in Muslim as well as Christian traditions and was recorded by pilgrims visiting the Holy Land on numerous occasions. The boiled water extracts of the leaves of $Z$. spina-christi are used by Muslims in the cleaning of a dead body before burial suggesting antibacterial properties. In addition, the plant has been used in mummification by the ancient Egyptians [1]. It has been suggested that the plant material referred to in the Bible as the "thorns" or "bramble" (Judges 9; 14-15), "thorns" (Matthew 27:27-29) and the "crown of thorn" (John 19:5) might have been derived from Z. spina-christi [2]. The Holy Quran mentions the Lote tree (Cedar) three times (XXXIV: 16; LIII: 13-18; LVI: 28-32), which was frequently identified as Z. spina-christi. Accordingly, this species is highly respected throughout the Middle East, has been widely used as a food and as medicinal as well as a environmental protection plant since antiquity, and is still in use at present $[2,3]$.

Z. spina-christi is commonly used in ethno-medicine for the treatment of many illnesses such as digestive disorders, weakness, hepatic disorders, obesity, urinary problems, diabetes, skin infections, fever, diarrhea and insomnia [4,5]. In Sudanese ethno-medicine, the leaves of $Z$. spina-christi are used for the treatment of malaria [6]. In addition, Michel et al. reported an anti-diabetic activity of the leaves of Z. spina-christi due to their saponin and polyphenol constituents [7], which was supported in pharmacological studies by Glombitza et al. indicating that extracts of $Z$. spina-christi leaves or its main saponin glycoside, christinin-A, enhanced glucose consumption in diabetic

*Corresponding author: Nikolai Kuhnert, Department of Life Science and Chemistry, Jacobs University Bremen, Campus Ring 8, 28759 Bremen, Germany, Tel: 494212003120; Fax: 494212003229; E-mail: n.kuhnert@jacobs-university.de

Received February 19, 2016; Accepted March 17, 2016; Published March 21 2016

Citation: Elsadig Karar MG, Quiet L, Rezk A, Jaiswal R, Rehders M, et al. (2016) Phenolic Profile and In Vitro Assessment of Cytotoxicity and Antibacterial Activity of Ziziphus spina-christi Leaf Extracts. Med chem (Los Angeles) 6: 143-156 doi:10.4172/2161-0444.1000339

Copyright: () 2016 Elsadig Karar MG, et al. This is an open-access article distributed under the terms of the Creative Commons Attribution License, which permits unrestricted use, distribution, and reproduction in any medium, provided the original author and source are credited. 
rats [8]. Furthermore, Z. spina-christi leaves and fruits are reported to possess antibacterial activity [9], as well as antifungal activity on plant pathogens [10]. In addition, Adzu et al. found that root bark extracts showed significant antinociceptive activity in mice and rats [11].

The widespread and incorrect use of antibacterial agents has caused emergence of bacterial strains that are resistant to several antibiotics. Bacteria have developed different defence strategies to protect themselves from antimicrobial drugs such as alteration of the drug target, enzymatic inactivation, reduction of intracellular drug concentration by modifications in membrane permeability, or by efflux pumps $[12,13]$. Accordingly, new antimicrobials with greater effectiveness and better tolerability than existing drugs are urgently required for treatment of bacterial infections. Plant extracts are wellknown to combine a diversity of phytoconstituents that have a broad spectrum of antimicrobial activities, consequently plant-derived bioactive compounds counted as the main source for lead compound identification and development in pharmaceutical production [14].

Plant polyphenols comprise a large group of secondary metabolites that can extend from simple molecules, such as phenolic acids, to highly polymerized compounds such as tannins. They are constituents with a rich number of derivatives in the plant kingdom, from mosses and ferns to higher angiosperms including food plants. Many of these plantderived polyphenols are essential components in our diets [15]. They are derivatives of the pentose phosphate, mevalonic acid, shikimate, and phenylpropanoid pathways in plants [16].

Flavonoids have been reported to have numerous biological effects such as antioxidant activity [17], antimicrobial activity [18], antiinflammatory activities [19], inhibition of platelet aggregation [20], and inhibition of mast cell histamine release [21]. Moreover, antioxidant phenolics have been suggested to possess preventive functions in the progression of heart diseases and different cancers including prostate, breast, lung, colon and rectal cancers [22]. In addition, epidemiological studies have demonstrated that there is an inverse relationship between the intake of flavonoids such as myricetin, apigenin, quercetin, kaempferol and luteolin, which are naturally present in fruits, vegetables and beverages, and the lowering of the risk of suffering from cardiovascular diseases [23].

From different species of Ziziphus, peptide and cyclopeptide alkaloids, flavonoids, sterols, tannins, betulinic acid and triterpenoidal saponin glycosides have been isolated and chemically identified $[24,25]$. From the methanol extract of the fruits of $Z$. jujube and Z. spina-christi twelve compounds have been reported as representing quercetin, kaempferol, and phloretin derivatives [15]. In a qualitative as well as quantitative study, flavonoids, saponins, and triterpenic acids were isolated from the leaves of two Ziziphus species [26].

Herein, the aim of the present work was to significantly improve and deepen our knowledge about the phenolic composition of $Z$. spina-christi leaves using liquid chromatography coupled to electron spray ionization multi-stage mass spectrometry (HPLC-ESI-MS ${ }^{n}$ ) as a powerful tool for the analysis of natural products and to evaluate the antimicrobial and cytotoxic effects of the plant extracts. The obtained results may contribute to a better understanding of the role of $Z$. spinachristi phenolics on biological, nutritional and medicinal properties.

\section{Methods}

\section{Chemicals and standards}

Procyanidin B1, Procyanidin B2, 5-O-caffeoylquinic acid, 3-O-caffeoylquinic acid and 4,5 di-O-caffeoylquinic acid were purchased from PhytoLab, Germany. Gallocatechin, epigallocatechin, catechin, epicatechin, kaempferol 3-O-glucoside, kaempferol 3-O-(6$O$-rhamnosyl-glucoside), quercetin 3-O-(6-O-rhamnosyl-glucoside) (rutin), quercetin 3-O-arabinoside, quercetin 3-O-rhamnoside, kaempferol-3-O-galactoside-rhamnoside-7-O-rhamnoside (robinin), quercetin 3-O-glucoside and the solvents methanol and DMSO were purchased from Sigma-Aldrich, Germany. Ultrapure water with a resistance of 18.2 M was deionized in a Milli-Q system (Sartorius Stedim Biotech, Germany).

\section{Plant material}

The Z. spina-christi plant samples were freshly collected in June 2010 from its natural habitats in Omdurman $\left(15^{\circ} 38^{\prime} 10^{\prime \prime}\right.$ North, $32^{\circ}$ 26'F 14" East), Khartoum, Sudan. The voucher specimen was identified by Dr. Hayder Abdel Gadir of Herbarium of Medicinal and Aromatic Plants Research Institute (MAPRI), Khartoum, Sudan, where the specimens (Voucher No. Zi-sp-ch-04) are maintained in a repository.

\section{Sample preparation}

Five grams of the shade-dried green leaves of the plants were ground in a hammer mill to fine powder and extracted with methanol/ water $(7: 3 \mathrm{v} / \mathrm{v})$ using a Soxhlet apparatus (Buchi Extraction System B-811/B-811 LSV, Germany) for $5 \mathrm{~h}$. These extracts were filtered through a Whatman no. 1 filter paper. The solvent was removed by evaporation in vacuo, and the extracts were stored in air-tight vial at $-20{ }^{\circ} \mathrm{C}$ until required for the photochemical analysis and cytotoxicity assays. For the photochemical analysis, the dried extract was thawed at room temperature, dissolved in methanol/water $(7: 3 \mathrm{v} / \mathrm{v} ; 60 \mathrm{mg} / 10 \mathrm{ml})$, filtered through a membrane filter with a pore size of $0.45 \mu \mathrm{m}$ (Carl Roth, Germany) and used directly for LC-MS.

For determining the antimicrobial activity, two additional methanol and aqueous extracts were prepared in a similar way. The methanol extract was concentrated to dryness using rotary evaporator in vacuo to obtain the crude methanol extract. The aqueous extract was concentrated and lyophilized. The respective extracts obtained were kept in air-tight vial at $-20^{\circ} \mathrm{C}$ until use.

\section{UV Irradiation}

UV irradiation experiments were performed as previously reported [27].

\section{Liquid chromatography-mass spectrometry (LC/ESI/MS ${ }^{\mathrm{n}}$ )}

The Agilent 1100 LC equipment (Agilent, Karlsruhe, Germany) comprised a binary pump, an auto-sampler with a $100-\mu \mathrm{l}$ loop, and a diode array detector with a light-pipe flow cell (recording at 254, 280, and $320 \mathrm{~nm}$ ). This was interfaced with an ion-trap mass spectrometer fitted with an electrospray ionization source (Bruker Daltonics HCT Ultra, Bremen, Germany) operating in full-scan, auto-MS ${ }^{\mathrm{n}}$ mode to obtain fragment ion $\mathrm{m} / z$. Tandem mass spectra were acquired in auto$\mathrm{MS}^{\mathrm{n}}$ mode (smart fragmentation) using a ramping of the collision energy. Maximum fragmentation amplitude was set to $1 \mathrm{~V}$, starting at $30 \%$ and ending at $200 \%$. MS operating conditions (negative mode) had been optimized using procyanidin $\mathrm{B} 1$ and 5-O-caffeoylquinic acid with a capillary temperature of $365^{\circ} \mathrm{C}$, a dry gas flow rate of $10 \mathrm{l} / \mathrm{min}$, and a nebulizer pressure of $10 \mathrm{psi}$. The spectra full scan mass were performed within the range from $\mathrm{m} / \mathrm{z} 50$ up to 1500 in negative ion mode.

High-resolution LC-MS was performed using the same high performance LC equipped with a micrOTOF mass spectrometer (Bruker Daltonics, Bremen, Germany) fitted with an electrospray ionization source, and internal calibration was achieved with $10 \mathrm{ml}$ of $0.1 \mathrm{M}$ sodium formate solution injected through a six-port valve prior 
to each chromatographic run. Calibration was performed using the enhanced quadratic mode.

\section{High-performance liquid chromatography}

The HPLC separation was were performed as previously reported [28].

\section{Preparative-HPLC isolation}

Preparative-HPLC isolation of compounds 26, 27, 39 and 50 was carried out as in our previous study [29].

\section{Antibacterial activity assay}

Three Gram-positive bacterial strains (Bacillus subtilis S168, Bacillus aquimaris MB-2011, and Clavibacter michiganensis GSPB 390 as well as three Gram-negative bacterial strains (Escherichia coli DH5a, Erwinia amylovora 1189, and Pseudomonas syringae pv tomato DC300) were selected as model organisms to evaluate the antibacterial activity of the methanol and aqueous $Z$. spina-christi crude plant extracts at a final concentrations of $100 \mathrm{mg} / \mathrm{ml}$. The antimicrobial activity assay was performed using the agar diffusion method according to Nathan et al. with slight modification as follows: Lysogeny broth (LB) agar plates were inoculated with $200 \mu \mathrm{l}$ of the tester organism $\left(1 \times 10^{7} \mathrm{cfu} / \mathrm{ml}\right)$ by spreading the bacterial cell suspensions over the agar with the help of sterile glass beads. Holes ( $5 \mathrm{~mm}$ diameter) were punched into the agar with a sterile Pasteur pipette, and $50 \mu$ of the crude extracts was pipetted into the wells [30]. The plates were incubated overnight at $28^{\circ} \mathrm{C}$ except for E. coli, for which incubation was done at $37^{\circ} \mathrm{C}$. Inhibition of microbial growth was determined by measuring the radius of the zone of inhibition. For each bacterial strain, an equal volume of water and methanol was used as negative controls. As positive controls, the following antibiotics were tested effective against the following bacterial organism causing inhibition zones of 10.3 to $23.3 \mathrm{~mm}$ : 25 $\mathrm{mg} / \mathrm{ml}$ kanamycin (grad $\geq 750 \mathrm{IU} / \mathrm{mg}$; Carl Roth, Germany) for $B$. subtilis and B. aquimaris; $50 \mathrm{mg} / \mathrm{ml}$ ampicillin (Carl Roth, Germany) for E. coli and E. amylovora; $25 \mathrm{mg} / \mathrm{ml}$ streptomycin (grade $730 \mathrm{IU} / \mathrm{mg}$; Serva, Germany) for P. syringae and C. michiganensis. The experiments were conducted in triplicates, and the data are given as mean values \pm standard deviation (SD). Each mean value was calculated from three biological replicas and each biological replica was calculated from three technical replicas. The SD was calculated from the mean of the three biological replicas. Data were analyzed with one-way analysis of variance (ANOVA) using Origin lab software.

\section{Cytotoxicity Analysis}

\section{Cell culture}

$\mathrm{HaCaT}$ cells at passages 40 - 55 were used as a model for epidermal keratinocytes [31,32]. They were cultured in Dulbecco's Modified Eagle Medium (DMEM, DMEM, Lonza Biowhittaker, Switzerland) containing phenol red and supplemented with $10 \%$ fetal bovine serum (FBS; Lonza Biowhittaker). The IEC- 6 cell line at passage 16 - 40 was used as cellular model of small intestine epithelial cells (European Collection of Cell Cultures, ECACC, UK), and cultured in DMEM containing $0.1 \mu \mathrm{g} / \mathrm{ml}$ insulin (Sigma-Aldrich, USA) and $5 \%$ FBS. Cells were grown at $37{ }^{\circ} \mathrm{C}$ in a humified atmosphere at $8 \% \mathrm{CO}_{2}$ for keratinocytes and at $5 \% \mathrm{CO}_{2}$ for IEC- 6 cells. Cell culture medium was exchanged every three to four days, confluent cell cultures were used for experimentation.

\section{Cellular integrity analyses by acquisition of propidium iodide (PI) and counter-staining with Draq $5^{\mathrm{mm}}$}

PI (Carl Roth, Germany) acquisition through ruptured plasma membranes was used as a measure of necrotic cell death, while Draq $5^{\mathrm{ma}}$ staining of cellular DNA served to determine total cell numbers [33]. Cells were grown on cover slips for 24 hours and then treated with $50 \mu \mathrm{l}$ of the plant extract at final concentrations of $100 \mu \mathrm{g} / \mathrm{ml}, 10 \mu \mathrm{g} / \mathrm{ml}$, and $1 \mu \mathrm{g} / \mathrm{ml}$ (weight/volume, final concentrations). Controls were treated with $0.5 \%$ DMSO, since all extracts were diluted with DMSO to this final concentration. After incubation of the cells with extract preparations for 24 hours at $37^{\circ} \mathrm{C}$, cells were washed with prewarmed PBS. Staining with $2 \mu \mathrm{g} / \mu \mathrm{l}$ PI (Carl Roth, Germany) diluted in the respective culture medium was for 30 minutes at $37^{\circ} \mathrm{C}$. Afterwards cells were rinsed with PBS twice before fixation with $4 \%$ paraformaldehyde (Applichem, Germany) in $200 \mathrm{mM}$ Hepes, pH 7.4 for 20 minutes at RT. Then, cells were rinsed in PBS and stained with $5 \mu \mathrm{M}$ Draq5 $5^{\mathrm{mx}}$ (Biostatus, UK) in PBS for 45 minutes at $37^{\circ} \mathrm{C}$. Finally, cells were washed three times with PBS and then mounted in mowiol (33\% glycerol and 14\% Mowiol (Carl Roth, Germany) in $200 \mathrm{nM}$ Tris-HCL in ultrapure $\mathrm{H}_{2} \mathrm{O}$ at $\mathrm{pH}$ 8.5).

Images were taken at a Zeiss LSM 510 Meta confocal laser scanning microscope (Carl Zeiss, Germany) and analyzed using Zeiss LSM Image Browser software version 3.2.0.70.

\section{Staining of the actin cytoskeleton with FITC-Phalloidin and counter-staining with Draq $5^{\mathrm{m}}$}

Cells were fixed as described above and stained with $3 \mu \mathrm{M}$ FITCPhalloidin (Sigma-Aldrich, Germany) and $5 \mu \mathrm{M}$ Draq $5^{\mathrm{Tn}}$ in PBS for 45 minutes at $37^{\circ} \mathrm{C}$ [34]. Cells were washed three times before mounting in mowiol.

\section{Determination of cell counts using the Image Analysis Software CellProfiler ${ }^{\mathrm{m}}$}

Images were analyzed using Cell Profiler 2.0 [35] as previously described [31,33,34].

\section{MTT Assay}

Cells were grown for 24 hours at $37{ }^{\circ} \mathrm{C}$ and the respective $\mathrm{CO}_{2}$ concentration in 24-well plates before treatment with $50 \mu \mathrm{l}$ plant extracts per $1 \mathrm{ml}$ culture medium to achieve final concentrations of the extracts to reach 100,10 and $1 \mu \mathrm{g} / \mathrm{ml}$ (wt/vol), respectively. As controls, cells were treated with $0.5 \%$ DMSO and mock-treated cells were used, to which no extracts were applied. MTT ([3-(4, 5-Dimethylthiazol-2yl)-2, 5-diphenyltetrazolium bromide; Carl Roth, Germany) was applied in PBS to the respective cell culture medium at a final concentration of $0.5 \mathrm{mg} / \mathrm{ml}$ MTT. Cells were incubated in the dark for 1.5 hours at $37^{\circ} \mathrm{C}$ and the respective $\mathrm{CO}_{2}$ concentration, before formazan crystals were dissolved in $500 \mu \mathrm{l}$ of $100 \%$ DMSO and quantified at $570 \mathrm{~nm}$ using a Thermo Spectronic Genesys 10 UV-vis Spectrophotometer (Thermo Scientific ${ }^{\mathrm{TN}}$, USA).

\section{Results and Discussion}

Methanol/water $(7: 3 \mathrm{v} / \mathrm{v})$ extract of the leaves of Z. spina-christi was separated by reversed-phase chromatography. Identification of compounds was obtained using high resolution-electrospray ionizationmass spectrometry (HR-ESI-MS) for determination of molecular formula and ESI with quadruple ion trap analyzer MS (ESI-QIT-MS) to obtain tandem MS data for further structure elucidation. Additionally, retention times and UV $(320 \mathrm{~nm})$ spectra were used for reversed phase compounds identification (Figure 1). Identification of phenolic compounds, for which commercial standards were available was carried out by the comparison of their retention times, UV-vis spectra and mass spectral data recorded in negative ion mode, while, the identity of other compounds was elucidated using the UV-vis spectrum to assign the phenolic class [36]. For all compounds the high resolution mass data were in good agreement with the theoretical molecular formulas 

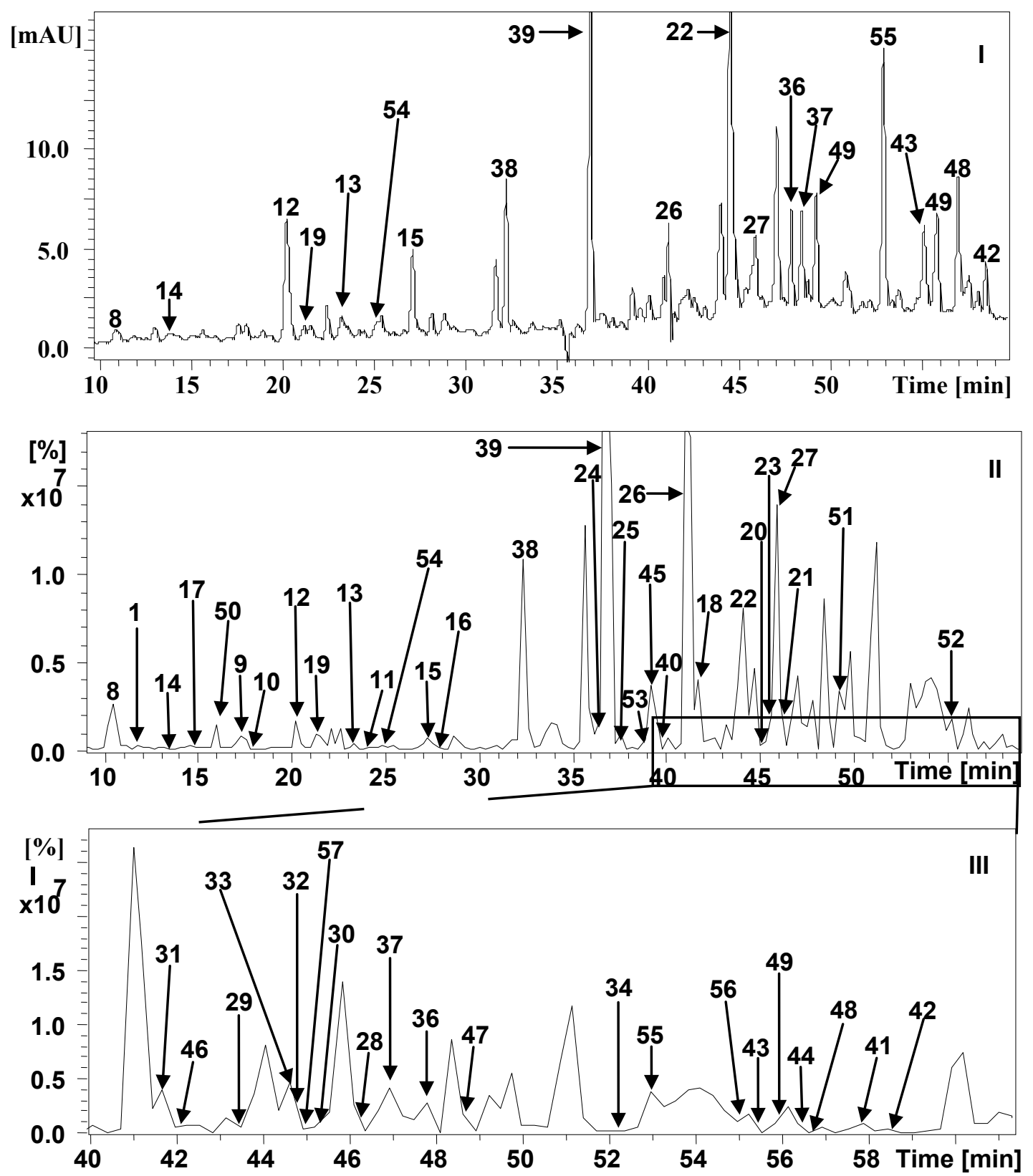

Figure 1: UV Chromatogram (320 nm) (I) base peak chromatogram (II) and expansion (III) for Z. spina-christi leaves extract. Peaks assignment listed in Table 1.

(Table 1S, Supporting information), all displaying a mass error of below $5 \mathrm{ppm}$, thus confirming their elemental composition. Moreover, the chromatographic elution order helped in some structural assignments as it was described previously [37]. The phytoconstituents characterized in Z. spina-christi are presented in Table 1 and their chemical structures are shown in Figure 2.

In the following section structure assignment of selected compounds is illustrated. Full assignment arguments are provided in the Supporting information.

\section{Characterization of Proanthocyanidins}

(Epi)catechin- $(4,8)$-(epi)gallocatechin (m/z 593)

One peak $(\mathrm{m} / \mathrm{z} 593)$ was detected at retention time $11.1 \mathrm{~min}$ in the extracted ion chromatogram (EIC) and was tentatively assigned as dimeric B-type proanthocyanidins with (epi)catechin and (epi) gallocatechin monomeric units (epi)catechin-(4, 8')-(epi)gallocatechin 1 (Table 1 and Figures $1 \mathrm{~S}$ and 2S, Supporting information) as evident from tandem MS data previously reported [38].

(Epi)gallocatechin- $\left(4,8^{\prime}\right)$-(epi)gallocatechin and (epi) gallocatechin-(4,6')-(epi)gallocatechin (m/z 609)

Six isomers $\left(\mathrm{t}_{\mathrm{R}} 6.0,7.7,9.6,11.1,11.9,14.8 \mathrm{~min}\right)$ were detected at $\mathrm{m} / \mathrm{z}$ 609 in the methanol/water extract of leaves of Z. spina-christi (Figures $3 \mathrm{~S}$ and $4 \mathrm{~S}$, Supporting information). Isomer 2-5 were assigned as (Epi) gallocatechin-(4, 8')-(epi)gallocatechin as previously reported [39]. While, isomers 6-7 were tentatively assigned as (epi)gallocatechin-(4, 6 ')-(epi)gallocatechin assuming that the latter are less polar and hence 
Citation: Elsadig Karar MG, Quiet L, Rezk A, Jaiswal R, Rehders M, et al. (2016) Phenolic Profile and In Vitro Assessment of Cytotoxicity and Antibacterial Activity of Ziziphus spina-christi Leaf Extracts. Med chem (Los Angeles) 6: 143-156. doi:10.4172/2161-0444.1000339

\begin{tabular}{|c|c|c|c|c|}
\hline No. & Compound name & $t_{R}(\min )$ & {$[\mathrm{M}-\mathrm{H}]^{-}$} & Characteristic $\mathrm{m} / \mathrm{z}$ of ions in negative ion mode \\
\hline 1 & (Epi)catechin-(4, 8')-(epi)gallocatechin & 11.1 & 593 & $\begin{array}{l}\mathrm{MS}^{2} \rightarrow 423(100), 305 \text { (46), 441(45), 467(25), 575(27); } \mathrm{MS}^{3} \rightarrow 297 \text { (100), 283(99), } 285 \text { (49), } 405 \\
(33)\end{array}$ \\
\hline 2 & (Epi)gallocatechin-(4, 8')-(epi)gallocatechin & 6.0 & 609 & $\begin{array}{l}\mathrm{MS}^{2} \rightarrow 423(100), 305 \text { (39), 441(75), } 483(15), 591(14) ; \mathrm{MS}^{3} \rightarrow 297(100), 283(85), 285(31) \\
405(15), 269(60), 255 \text { (33), } 243(34) ; \mathrm{MS}^{4} \rightarrow 297(100), 269(30)\end{array}$ \\
\hline 3 & (Epi)gallocatechin-(4, 8')-(epi)gallocatechin & 7.7 & 609 & $\begin{array}{l}\mathrm{MS}^{2} \rightarrow 423(100), 305 \text { (30), 441(81), } 483(11), 591(13) ; \mathrm{MS}^{3} \rightarrow 297(100), 283 \text { (91), 405(21), } 255 \\
(28), 243(23) ; \mathrm{MS}^{4} \rightarrow 297(100), 269(60)\end{array}$ \\
\hline 4 & (Epi)gallocatechin-(4, 6')-(epi)gallocatechin & 9.6 & 609 & $\begin{array}{l}\mathrm{MS}^{2} \rightarrow 441 \text { (100), } 423(99), 305 \text { (27), } 483 \text { (18), } 539 \text { (10); } \mathrm{MS}^{3} \rightarrow 283(100), 255(29), 297 \text { (81), } \\
405 \text { (16), } 243(21) ; \mathrm{MS}^{4} \rightarrow 297 \text { (100), } 269 \text { (68) }\end{array}$ \\
\hline 5 & (Epi)gallocatechin-(4, 8')-(epi)gallocatechin & 11.1 & 609 & $\begin{array}{l}\mathrm{MS}^{2} \rightarrow 423(100), 305(40), 441(70), 483(19), 591(07) ; \mathrm{MS}^{3} \rightarrow 297(100), 283(83), 405(07), 255 \\
(27), 269(23) ; S^{4} \rightarrow 297 \text { (100), } 269 \text { (42), } 253(16)\end{array}$ \\
\hline 6 & (Epi)gallocatechin-(4, 6')-(epi)gallocatechin & 11.9 & 609 & $\begin{array}{l}\mathrm{MS}^{2} \rightarrow 423(100), 305(36), 441(76), 483(14), 593(08), 591(13) ; \mathrm{MS}^{3} \rightarrow 283(100), 297(67) \\
255(24), 405(22), 255(24), 269(14), 243(34) ; \mathrm{MS}^{4} \rightarrow 283(100), 255(30)\end{array}$ \\
\hline 7 & (Epi)gallocatechin-(4, 8')-(epi)gallocatechin & 14.8 & 609 & $\begin{array}{l}\mathrm{MS}^{2} \rightarrow 423(100), 305(29), 441(66), 483(14), 539(10), 591(10) ; \mathrm{MS}^{3} \rightarrow 283(100), 297(89) \\
405(12), 243(34) ; \mathrm{MS}^{4} \rightarrow 297(100), 269 \text { (99), } 255 \text { (34) }\end{array}$ \\
\hline 8 & Gallocatechin & 10.5 & 305 & $\begin{array}{l}\mathrm{MS}^{2} \rightarrow 179(100), 219(69), 261(10), 165(26), 137(22), 125(36) ; \mathrm{MS}^{3} \rightarrow 164(100), 151(27) \\
135(36), 243(34) ; \mathrm{MS}^{4} \rightarrow 120(100)\end{array}$ \\
\hline 9 & Epigallocatechin & 17.3 & 305 & $\begin{array}{l}\mathrm{MS}^{2} \rightarrow 179(100), 219(92), 261(28), 165(24), 137(26), 125(36) ; \mathrm{MS}^{3} \rightarrow 164(100), 151(14) \\
\text { 135(23), } 247(16)\end{array}$ \\
\hline 10 & Catechin & 17.8 & 289 & $\begin{array}{l}\mathrm{MS}^{2} \rightarrow 244(100), 205(28) ; \mathrm{MS}^{3} \rightarrow 203\left(100,227(18), 186(16), 161(20) ; \mathrm{MS}^{4} \rightarrow 174(100), 187\right. \\
(70)\end{array}$ \\
\hline 11 & Epicatechin & 24.0 & 289 & $\begin{array}{l}\mathrm{MS}^{2} \rightarrow 244(100), 205 \text { (40); } \mathrm{MS}^{3} \rightarrow 03 \text { (100), 227(25), 186(16), } 161 \text { (21); MS } \rightarrow 174 \text { (100), } 187 \\
(1)\end{array}$ \\
\hline 12 & trans-5-O-Caffeoylquinic acid & 20.2 & 353 & $\mathrm{MS}^{2} \rightarrow 191(100) ; \mathrm{MS}^{3} \rightarrow 127(10), 173(75), 85(73) ; \mathrm{MS}^{4} \rightarrow 109(100)$ \\
\hline 13 & cis-5-O-Caffeoylquinic acid & 23.2 & 353 & $\mathrm{MS}^{2} \rightarrow 191$ (100), 179 (40), 135 (11); MS $\rightarrow 127$ (100), 111 (55), 85 (41), 59 (18) \\
\hline 14 & 3-O-Caffeoylquinic acid & 13.5 & 353 & $\mathrm{MS}^{2} \rightarrow 191(100) ; \mathrm{MS}^{3} \rightarrow 111(100), 173(85), 127(45), 85$ (33) \\
\hline 15 & trans-5-O-p-Coumaroylquinic acid & 27.2 & 337 & $\mathrm{MS}^{2} \rightarrow 191(100) ; \mathrm{MS}^{3} \rightarrow 127(100), 173(47), 85(92)$ \\
\hline 16 & cis-5-O-p-Coumaroylquinic acid & 28.0 & 337 & $\mathrm{MS}^{2} \rightarrow 191(100) ; \mathrm{MS}^{3} \rightarrow 127(100), 173(51), 85(71)$ \\
\hline 17 & Caffeic acid 4-O-glucoside & 14.9 & 341 & $\mathrm{MS}^{2} \rightarrow 179$ (100), $161(26), 135(15) ; \mathrm{MS}^{3} \rightarrow 135$ (100) \\
\hline 18 & 4, 5-di-O-Caffeoylquinic acid & 41.7 & 515 & MS $^{2} \rightarrow 353$ (100), 203 (17), 173 (6), $179(7) ;$ MS $^{3} \rightarrow 123$ (100), 179(23), 191 (19), 135 (7) \\
\hline 19 & Apigenin 7, 4'-di-O-glucoside & 21.4 & 593 & $\mathrm{MS}^{2} \rightarrow 431(100) ; \mathrm{MS}^{3} \rightarrow 269(100), 161(45)$ \\
\hline 20 & $\begin{array}{l}\text { Isorhamnetin 3-O-(6-O-rhamnosyl- } \\
\text { galactoside) }\end{array}$ & 45.1 & 623 & $\mathrm{MS}^{2} \rightarrow 315$ (100), 300 (27), $271(14) ; \mathrm{MS}^{3} \rightarrow 300$ (100); $\mathrm{MS}^{4} \rightarrow 271$ (100), 255 (53) \\
\hline 21 & $\begin{array}{l}\text { Isorhamnetin 3-O-(6-O-rhamnosyl- } \\
\text { glucoside) }\end{array}$ & 46.0 & 623 & $\mathrm{MS}^{2} \rightarrow 315$ (100), 300 (15), $271(11) ; \mathrm{MS}^{3} \rightarrow 300$ (100); MS $\rightarrow 271(100), 255(8)$ \\
\hline 22 & $\begin{array}{l}\text { Kaempferol 3-O-(6-O-rhamnosyl- } \\
\text { galactoside) }\end{array}$ & 44.0 & 593 & $\begin{array}{l}\mathrm{MS}^{2} \rightarrow 285(100) 284(55) ; \mathrm{MS}^{3} \rightarrow 255(100), 257 \text { (33), } 229 \text { (12), } 241(13), 151(10), \mathrm{MS}^{4} \rightarrow 255 \\
(100), 229 \text { (34), } 163(46)\end{array}$ \\
\hline 23 & Kaempferol 3-O-(6-O-rhamnosyl-glucoside) & 45.5 & 593 & $\begin{array}{l}\mathrm{MS}^{2} \rightarrow 285(100) ; \mathrm{MS}^{3} \rightarrow 257(100), 229(38), 241(29), 255(18), 163(26), \mathrm{MS}^{4} \rightarrow 255(100) \\
229(58), 163(66)\end{array}$ \\
\hline 24 & Quercetin 7-O-(6-O-rhamnosyl-glucoside) & 36.4 & 609 & $\begin{array}{l}\mathrm{MS}^{2} \rightarrow 300(100), 301(32), 271(20), 255(10), 445 \text { (10), } 489(11) ; \mathrm{MS}^{3} \rightarrow 271(100), 179(14) \\
255 \text { (59); } \mathrm{MS}^{4} \rightarrow 271(100), 255(34), 243(49)\end{array}$ \\
\hline 25 & Quercetin 3-O-(6-O-rhamnosyl-galactoside) & 37.3 & 609 & $\begin{array}{l}\mathrm{MS}^{2} \rightarrow 300 \text { (100), 301(40), } 271(24), 255(12) ; \mathrm{MS}^{3} \rightarrow 300 \text { (100), } 271(77), 179(11), 255(41) \\
\mathrm{MS}^{4} \rightarrow 271(100)\end{array}$ \\
\hline 26 & $\begin{array}{l}\text { Quercetin 3-O-(6-O-rhamnosyl-glucoside) } \\
\text { (Rutin) }\end{array}$ & 41.0 & 609 & $\mathrm{MS}^{2} \rightarrow 300$ (100), 301(50), 271 (12); $\mathrm{MS}^{3} \rightarrow 179$ (100), 151 (72), 271 (63), 255 (38) \\
\hline 27 & Quercetin 3-O-(2-O-rhamnosyl-arabinoside) & 45.8 & 579 & $\mathrm{MS}^{2} \rightarrow 301$ (32), 300 (100), MS $\rightarrow 271$ (100), 255 (57); MS $\rightarrow 271$ (100), 255 (78), 243 (79) \\
\hline 28 & Quercetin 3-O-(2-O-rhamnosyl-xyloside) & 46.1 & 579 & $\begin{array}{l}\mathrm{MS}^{2} \rightarrow 300 \text { (100), } 301(28), 271(18), \mathrm{MS}^{3} \rightarrow 271(100), 255(57), 179(11), 151(10) ; \mathrm{MS}^{4} \rightarrow 255 \\
(100), 271(41), 244(18), 227(44), 215(32), 199(38), 151(16)\end{array}$ \\
\hline 29 & Quercetin 3-O-arabinoside & 43.4 & 433 & $\mathrm{MS}^{2} \rightarrow 301$ (100), 300 (63), 377 (17); MS $\rightarrow 271$ (100), 255 (49), 179 (68), 151 (53) \\
\hline 30 & Quercetin 3-O-rhamnoside & 45.2 & 447 & $\mathrm{MS}^{2} \rightarrow 301$ (100); MS $\rightarrow 179$ (100), 271 (42), 255 (24), $151(56), \mathrm{MS}^{4} \rightarrow 151(100)$ \\
\hline 31 & Quercetin 3-O-glucoside & 41.7 & 463 & $\mathrm{MS}^{2} \rightarrow 301$ (100), $343(10) ; \mathrm{MS}^{3} \rightarrow 179$ (100), 151 (59), 255 (18), $271(30) ; \mathrm{MS}^{4} \rightarrow 151(100)$ \\
\hline 32 & Quercetin -O-acetyl hexoside & 45.2 & 505 & $\begin{array}{l}\mathrm{MS}^{2} \rightarrow 463 \text { (100), } 301(34), 447(64) ; \mathrm{MS}^{3} \rightarrow 327 \text { (100), } 301 \text { (9), } 271 \text { (9), } 257 \text { (6); } \mathrm{MS}^{4} \rightarrow \\
\text { 223(100), } 271 \text { (53), } 241(21)\end{array}$ \\
\hline 33 & Kaempferol 3-O-glucoside & 44.6 & 447 & $\begin{array}{l}\mathrm{MS}^{2} \rightarrow 284 \text { (100), } 285 \text { (58), } 255 \text { (22), } 327 \text { (13); MS } \rightarrow 255 \text { (100); MS } \rightarrow 227 \text { (100), } 211 \text { (39), } \\
163(19)\end{array}$ \\
\hline 34 & Kaempferol 3-O-rhamnoside & 50.2 & 431 & $\mathrm{MS}^{2} \rightarrow 284$ (100), 285 (88), 255 (12); MS $\rightarrow 255$ (100), 267 (16), 229 (13) \\
\hline 35 & $\begin{array}{l}\text { Quercetin 3-O-[6"-(3-hydroxyl-3- } \\
\text { methylglutaryl)- } \beta-D-\text { galactoside] }\end{array}$ & 43.1 & 607 & $\begin{array}{l}\mathrm{MS}^{2} \rightarrow 463 \text { (100), } 301(11), 505(41), 545(18) ; \mathrm{MS}^{3} \rightarrow 301(100) ; \mathrm{MS}^{4} \rightarrow 179(100), 271(24) \\
255 \text { (15), } 151 \text { (56), } 151(71)\end{array}$ \\
\hline 36 & $\begin{array}{l}\text { kaempferol 3-O-[6"-(3-hydroxyl-3- } \\
\text { methylglutaryl)- } \beta-D-\text { galactoside] }\end{array}$ & 47.8 & 591 & $\begin{array}{l}\mathrm{MS}^{2} \rightarrow 447(100), 285(25), 489(50), 523(31), 529(45) ; \mathrm{MS}^{3} \rightarrow 285(100) ; \mathrm{MS}^{4} \rightarrow 257(100) \\
267(87), 239(37), 163(37)\end{array}$ \\
\hline 37 & $\begin{array}{l}\text { Quercetin 3-O-ß-D-xylosyl-(1 } \rightarrow 2)-\alpha-L- \\
\text { rhamnoside-4'-O- } \alpha \text {-L-rhamnoside }\end{array}$ & 46.9 & 725 & $\begin{array}{l}\mathrm{MS}^{2} \rightarrow 579(100) ; \mathrm{MS}^{3} \rightarrow 300(100), 301(27), 447(12), 271(22), 255(12), \mathrm{MS}^{4} \rightarrow 271(100), 255 \\
(46)\end{array}$ \\
\hline 38 & Phloretin 3', 5'-di-C-galactoside & 32.6 & 597 & $\begin{array}{l}\mathrm{MS}^{2} \rightarrow 357(100), 387(66), 417(18), 477(18) ; \mathrm{MS}^{3} \rightarrow 209(100), 251(11), 167(7), 123(7) ; \mathrm{MS}^{4} \\
\rightarrow 123(100), 165(82), 191(26), 93(29)\end{array}$ \\
\hline 39 & $\begin{array}{l}\text { Phloretin 3'-C-glucoside } 5^{\prime}-C \text {-galactoside or } \\
\text { phloretin } 3^{\prime}-C \text {-galactoside } 5^{\prime}-C \text {-glucoside }\end{array}$ & 36.8 & 597 & $\begin{array}{l}\mathrm{MS}^{2} \rightarrow 357(100), 387(65), 417(17), 477(53) ; \mathrm{MS}^{3} \rightarrow 209(100), 251(12), 167(9), 123(5) ; \mathrm{MS}^{4} \\
\rightarrow 123(100), 165 \text { (45), } 191(18)\end{array}$ \\
\hline 40 & Phloretin 3', 5'-di-C-glucoside & 39.4 & 597 & $\begin{array}{l}\mathrm{MS}^{2} \rightarrow 357(100), 387(66), 417(22), 477(42) ; \mathrm{MS}^{3} \rightarrow 209(100), 251(12), 167(8), 123(7) ; \mathrm{MS}^{4} \\
\rightarrow 123(100), 165(34), 191(14)\end{array}$ \\
\hline
\end{tabular}




\begin{tabular}{|c|c|c|c|c|}
\hline 41 & $\begin{array}{l}\text { Quercetin 3-O-(2, 6-di-O-rhamnosyl- } \\
\text { galactoside) 7-di-O-rhamnoside }\end{array}$ & 57.5 & 1047 & $\begin{array}{l}\mathrm{MS}^{2} \rightarrow 901(100), 755(60) ; \mathrm{MS}^{3} \rightarrow 755(100), 781(14) ; \mathrm{MS}^{4} \rightarrow 300(100), 301(56), 271(26) \\
255(14), 609(15), 489(17), 591(16)\end{array}$ \\
\hline 42 & $\begin{array}{l}\text { Quercetin 3-O-(2, 6-di-O-rhamnosyl- } \\
\text { glucoside) 7-di-O-rhamnoside }\end{array}$ & 58.4 & 1047 & $\begin{array}{l}\mathrm{MS}^{2} \rightarrow 901(100), 755 \text { (72), } 781(22) ; \mathrm{MS}^{3} \rightarrow 755(100), 781(10) ; \mathrm{MS}^{4} \rightarrow 300 \text { (100), } 301 \text { (47), } \\
271(19), 255 \text { (15), } 343(15), 609(28), 489(30), 591(21)\end{array}$ \\
\hline 43 & Diosmetin 3'-O-galactoside 7-O-rutinoside & 54.4 & 769 & $\begin{array}{l}\mathrm{MS}^{2} \rightarrow 607(100), 469 \text { (20), } 733 \text { (10), } 714 \text { (73), } 469 \text { (22), 299(31), } 285 \text { (20); MS } \rightarrow 299 \text { (100), } \\
255 \text { (29), } 284(43)\end{array}$ \\
\hline 44 & Diosmetin 3'-O-glucoside 7-O-rutinoside & 56.6 & 769 & $\begin{array}{l}\mathrm{MS}^{2} \rightarrow 607 \text { (100), } 469 \text { (23), } 733 \text { (81), } 714 \text { (73), } 453 \text { (55), } 315 \text { (76), } 299 \text { (60), } 285 \text { (37); MS } \rightarrow \\
299 \text { (100), } 255 \text { (56) }\end{array}$ \\
\hline 45 & $\begin{array}{l}\text { Kaempferol 3-O-(2, 6-di-O-rhamnosyl- } \\
\text { glucoside) }\end{array}$ & 39.2 & 739 & $\begin{array}{l}\text { MS }^{2} \rightarrow 285(100), 284 \text { (60), } 255 \text { (42), } 575 \text { (76), } 593 \text { (29), } 473 \text { (13), } 327 \text { (23), } 393 \text { (45); } \text { MS }^{3} \rightarrow \\
284 \text { (100), } 285 \text { (14), } 255 \text { (29), } 151 \text { (15); MS } \rightarrow 255 \text { (100) }\end{array}$ \\
\hline 46 & $\begin{array}{l}\text { Quercetin 3-O-(2, 6-di-O-rhamnosyl- } \\
\text { glucoside) } 7 \text {-O-galactoside }\end{array}$ & 42.2 & 917 & $\begin{array}{l}\mathrm{MS}^{2} \rightarrow 755(100) ; \mathrm{MS}^{3} \rightarrow 300(100), 301 \text { (50), } 271 \text { (29), } 255 \text { (12), } 343(22), 609 \text { (11), } 591 \text { (29); } \\
\mathrm{MS}^{4} \rightarrow 271(100), 256(50), 179(20), 151(17)\end{array}$ \\
\hline 47 & $\begin{array}{l}\text { Quercetin 3-O-(2, 6-di-O-rhamnosyl- } \\
\text { glucoside) 7-O-glucoside }\end{array}$ & 48.9 & 917 & $\begin{array}{l}\mathrm{MS}^{2} \rightarrow 755(100) ; \mathrm{MS}^{3} \rightarrow 300(100), 301(42), 271(12), 255 \text { (12), } 591 \text { (34), } 489 \text { (18), } 325 \text { (21); } \\
\mathrm{MS}^{4} \rightarrow 284 \text { (100), } 271 \text { (95), } 255 \text { (26), } 243(20), 169(12)\end{array}$ \\
\hline 48 & Kaempferol 3 -O-rutinoside 7-O-rhamnoside & 56.6 & 739 & $\mathrm{MS}^{2} \rightarrow 593$ (100); $\mathrm{MS}^{3} \rightarrow 285$ (100), 284 (89), 429 (39); MS $\rightarrow 257$ (100), 255 (32) \\
\hline 49 & $\begin{array}{l}\text { Kaempferol 3-O-(2, 6-di-O-rhamnosyl- } \\
\text { glucoside) 7-O-ramnoside }\end{array}$ & 55.8 & 885 & $\begin{array}{l}\mathrm{MS}^{2} \rightarrow 739(100), 285(25) ; \mathrm{MS}^{3} \rightarrow 285 \text { (100), } 284 \text { (69), } 255 \text { (33), } 575 \text { (75), } 473 \text { (18), } 393 \text { (45), } \\
327 \text { (33); } \mathrm{MS}^{4} \rightarrow 255 \text { (100), } 241 \text { (17), } 151 \text { (42) }\end{array}$ \\
\hline 50 & $\begin{array}{l}\text { Quercetin 3-O-(2, 6-di-O-rhamnosyl- } \\
\text { glucoside) }\end{array}$ & 15.6 & 755 & $\begin{array}{l}\mathrm{MS}^{2} \rightarrow 300(100), 301(17), 271(19), 255(9), 591(18), 609(13), 489(13), 343(19) ; \mathrm{MS}^{3} \rightarrow 271 \\
(100), 255 \text { (60), } 179 \text { (24), } 151 \text { (17); } \mathrm{MS}^{4} \rightarrow 255 \text { (100), } 271 \text { (83), } 227 \text { (37), } 243 \text { (99) }\end{array}$ \\
\hline 51 & $\begin{array}{l}\text { Quercetin 3-O-rhamnosyl-galactoside } \\
\text { 7-O-rhamnoside }\end{array}$ & 49.2 & 755 & $\begin{array}{l}\mathrm{MS}^{2} \rightarrow 609(100), 301(33) ; \mathrm{MS}^{3} \rightarrow 301(100), 300 \text { (51), } 271 \text { (12), } 343(15) ; \mathrm{MS}^{4} \rightarrow 179(100) \\
271 \text { (90), } 255 \text { (85), } 151 \text { (83) }\end{array}$ \\
\hline 52 & Quercetin 3-O-rutinoside 7-O-rhamnoside & 55.2 & 755 & $\begin{array}{l}\mathrm{MS}^{2} \rightarrow 609(100), 301(47) ; \mathrm{MS}^{3} \rightarrow 301(100), 300 \text { (51), } 343 \text { (12); } \mathrm{MS}^{4} \rightarrow 179 \text { (100), } 271 \text { (71), } \\
255 \text { (62), } 151 \text { (63) }\end{array}$ \\
\hline 53 & $\begin{array}{l}\text { Quercetin 3-O-(rhamnosyl-( }(\alpha \rightarrow 6)-O- \\
\text { glucoside) hexoside }\end{array}$ & 38.8 & 771 & $\mathrm{MS}^{2} \rightarrow 301$ (100), 609(7); $\mathrm{MS}^{3} \rightarrow 179(100), 151(84), 273(9) ; \mathrm{MS}^{4} \rightarrow 151$ (100), $169(10)$ \\
\hline 54 & Quercetin 3 -O-rutinoside 7-O-glucoside & 24.6 & 771 & $\begin{array}{l}\mathrm{MS}^{2} \rightarrow 609 \text { (100), } 301(5) ; \mathrm{MS}^{3} \rightarrow 301(100), 300 \text { (38), } 271 \text { (9), } 255 \text { (4); MS } \rightarrow 300 \text { (100), } 273 \\
(10), 255 \text { (6), } 179 \text { (29), } 151(12)\end{array}$ \\
\hline 55 & $\begin{array}{l}\text { Quercetin 3-O-(2, 6-di-O-rhamnosyl- } \\
\text { galactoside) 7-O-rhamnoside }\end{array}$ & 52.9 & 901 & $\begin{array}{l}\mathrm{MS}^{2} \rightarrow 755(100) ; \mathrm{MS}^{3} \rightarrow 300(100), 301(44), 271(30), 255 \text { (15), } 609 \text { (17), } 343(21) ; \mathrm{MS}^{4} \rightarrow 271 \\
(100), 255 \text { (48), } 179 \text { (14), } 151 \text { (18) }\end{array}$ \\
\hline 56 & $\begin{array}{l}\text { Quercetin 3-O-(2, 6-di-O-rhamnosyl- } \\
\text { glucoside) 7-O-rhamnoside }\end{array}$ & 54.9 & 901 & $\begin{array}{l}\mathrm{MS}^{2} \rightarrow 755(100) ; \mathrm{MS}^{3} \rightarrow 300(100), 301(60), 271(29), 255 \text { (13), } 343(25), 609 \text { (14), } 489(21) \\
\mathrm{MS}^{4} \rightarrow 271(100), 255(60), 179 \text { (33), } 151(25)\end{array}$ \\
\hline 57 & $\begin{array}{l}\text { Quercetin 3-O-(2, 6-di-O-rhamnosyl- } \\
\text { glucoside) 7-O-glucuronide }\end{array}$ & 44.9 & 931 & $\begin{array}{l}\mathrm{MS}^{2} \rightarrow 755(100), 769 \text { (65), } 781(17), 795(10) ; \mathrm{MS}^{3} \rightarrow 300 \text { (100), } 301 \text { (43), } 271 \text { (27), } 255 \text { (12); } \\
\mathrm{MS}^{4} \rightarrow 255 \text { (100), } 271 \text { (74), } 179 \text { (11), } 151(17)\end{array}$ \\
\hline
\end{tabular}

Table 1: Retention times $\left(\mathrm{t}_{\mathrm{R}}\right)$ and $\mathrm{MS}^{4}$ fragmentation of $Z$. spina-christi phenolics.

eluted later in the RP C18-Amid column (Table 1, Figures 1 and 2). (See also Figure 5S, Supporting information for the fragmentation pathway).

\section{Gallocatechin isomers $(\mathrm{m} / \mathrm{z} 305)$}

At retention times 10.5 and $17.3 \mathrm{~min}$, two peaks with $\mathrm{m} / \mathrm{z} 305$ were detected in the EIC and base peak chromatogram (BPC) and were assigned as gallocatechin 8 and epigallcatechin 9 (Table 1) after comparison with the retention time and fragmentation pattern of gallocatechin and epigallcatechin commercial standards.

\section{Catechin isomers $(\mathrm{m} / \mathrm{z} 289)$}

Two peaks ( $\mathrm{t}_{\mathrm{R}} 17.8$ and $\left.24.0 \mathrm{~min}\right)$ were detected at $\mathrm{m} / z 289$ in the EIC and were identified as catechin 10 and epicatechin 11 (Table 1) by comparison of their UV and mass spectra with authentic standards.

\section{Characterization of chlorogenic acids}

trans-5-O-caffeoylquinic acid 12 , trans-5-O-p-coumaroylquinic acid 15 and cis-5-O-p-coumaroylquinic acid 16 were easily detected in the EIC and total ion chromatogram TIC (Table 1), while 3-O-caffeoylquinic acid 14 at $m / z 353$, and 4, 5 di-O-caffeoylquinic acid 18 at $m / z 515$ were observed in low intensities and were not determined in the BPC or total ion chromatogram (TIC). Subsequently, targeted tandem LC-MS experiments at $m / z 353$ and $m / z 515$, respectively, have been performed to confirm there presence in the plant extract. The chlorogenic acids were assigned after the comparison with the retention time and fragmentation pattern of authentic standard supported with the hierarchical keys previously reported in the literature [40-43]. At retention time $23.2 \mathrm{~min}$, a further small peak $(\mathrm{m} / \mathrm{z}$ $353)$ with low intensity (16\%) compared to compound $12(100 \%)$ was observed. Targeted tandem-LC-MS experiments at $\mathrm{m} / \mathrm{z} 353$ have been performed and it displayed fragmentation patterns identical to the 5-O-caffeoylquinic acid and we suspected that it might be a cis isomer of the 5-O-caffeoylquinic. For confirmation of this isomer, the extract of $Z$. spina-christi was irradiated with UV light at $245 \mathrm{~nm}$ for $60 \mathrm{~min}$. After irradiation, we found that the cis isomer in the chromatogram as peak with considerably increased intensities if compared to trans isomer from the original plant extract (Figures 7S and 8S, Supporting information), which confirmed the presence of the cis-5-O-caffeoylquinic acids 13 [27].

\section{Characterization of caffeic acid 4-O-glucoside $(\mathrm{m} / \mathrm{z} 341)$}

One peak was readily detected at $\mathrm{m} / z 341$ in the EIC and was tentatively assigned as caffeic acid 4-O-glucoside 17 . The neutral loss of a glucosyl moiety $(162 \mathrm{u})$ resulted in a dominant fragment ion at $\mathrm{m} / \mathrm{z} 179.0$ corresponding to deprotonated caffeic acid and secondary peak at $m / z 161\left(\left[\mathrm{M}-\mathrm{H}-162-\mathrm{H}_{2} \mathrm{O}\right]^{-}\right)$from the neutral loss of a water molecule and an $\mathrm{MS}^{3}$ base peak at $\mathrm{m} / z 135$ by the neutral loss of $\mathrm{CO}_{2}$ $(44 \mathrm{u})$ (Figure 9S, Supporting information). For further evidence the UV spectrum, $\mathrm{MS}^{n}$ fragmentation and the retention time of this compound was compared with the compound $(\mathrm{m} / z$ 341) reported in the flowers of Chrysanthemum morifolium Ramat [44] and found that they are identical. Based on the above arguments compound 17 was identified as caffeic acid 4-O-glucoside. We have recently identified 6-O-caffeoyl- $\alpha$-glucose and 6-O-caffeoyl- $\beta$-glucose in the bottle gourd (Lagenaria siceraria Stand) fruits [45]. Here we are unable to assign the anomeric structure for the glucose moiety.

\section{Characterization of apigenin 7,4'-di-O-glucoside (m/z 593)}

One peak was detected at retention time 21.4 min with $\mathrm{m} / \mathrm{z} 593$ in the Z. spina-christi methanol/water extract. It showed fragmentation 
A

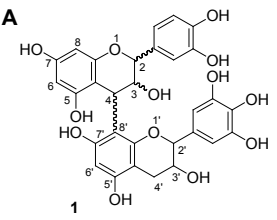<smiles>CC1c2ccc(O)cc2OC1c1cc(O)c(O)cc1O</smiles>

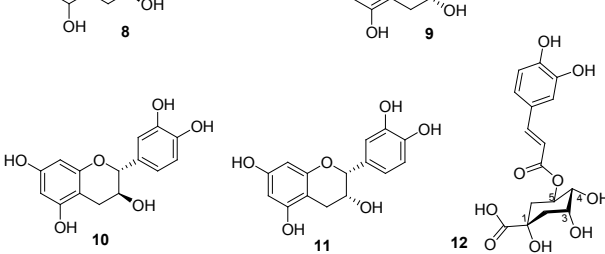<smiles></smiles>
13 O HO 

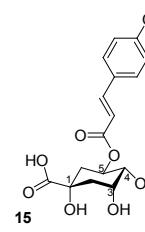

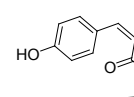$$
\text { }
$$<smiles>O=C(O)C(O)C(O)C(=O)OCC=Cc1cccc(O)c1</smiles>

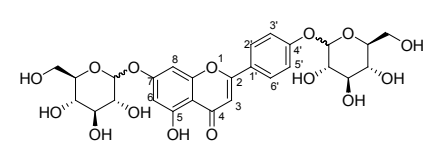

c

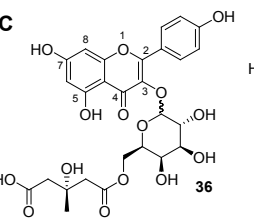

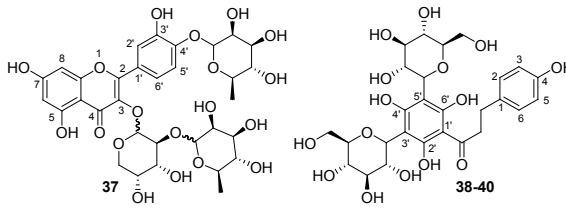

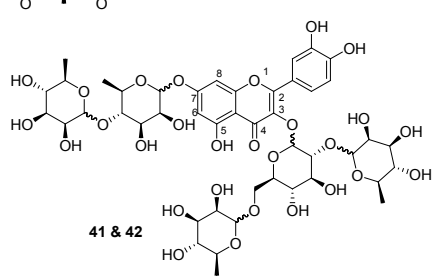

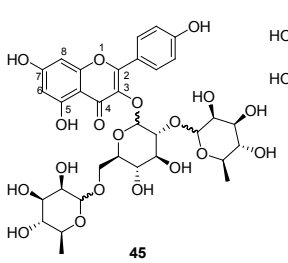<smiles></smiles>

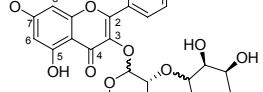

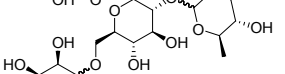

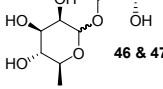
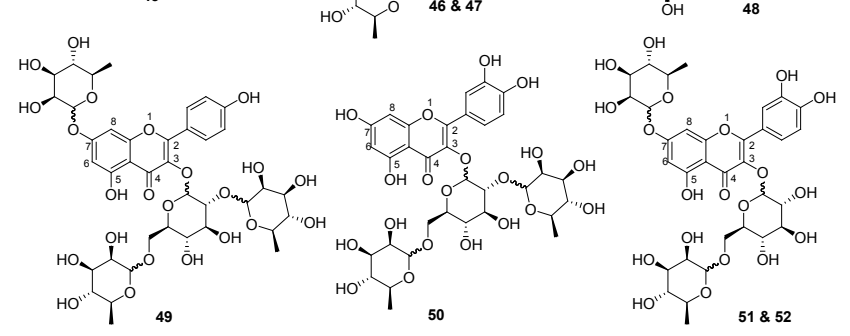

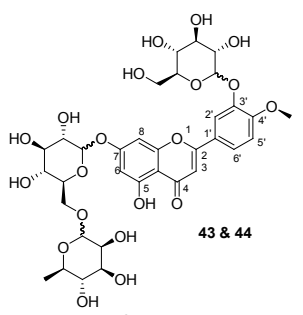

OH
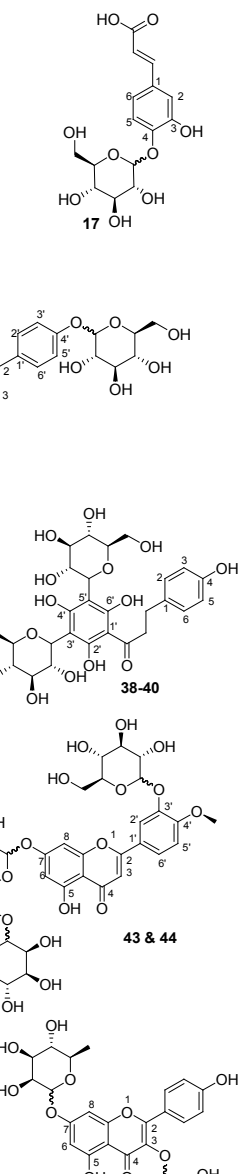

$\underbrace{\mathrm{O} H}_{\mathrm{OH}}$

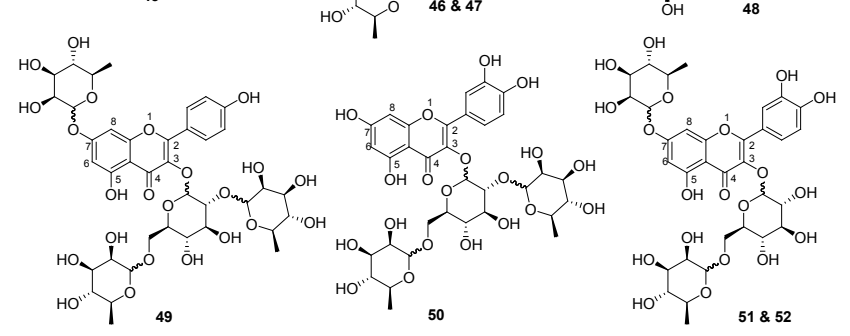

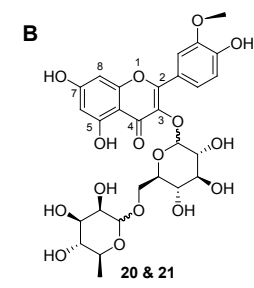
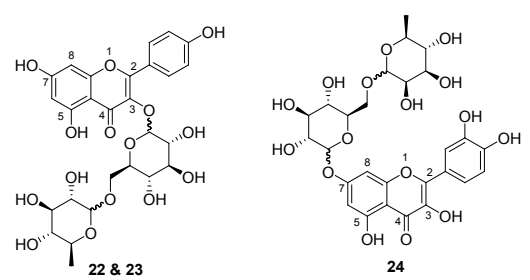

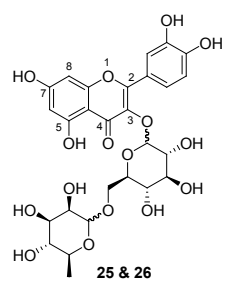

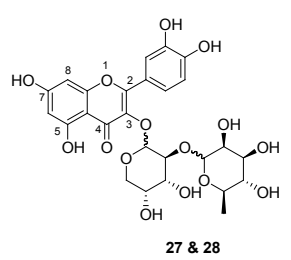

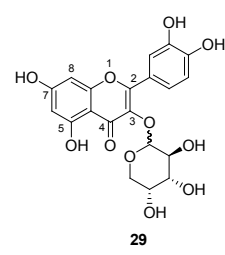

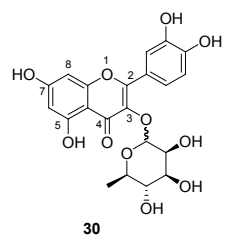

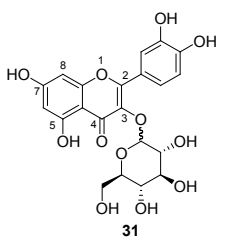

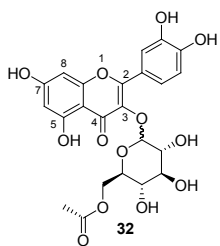<smiles>COc1c(C)cccc1C(=O)OC1C(O)C(O)C(O)C(O)C1c1ccc(O)cc1</smiles>

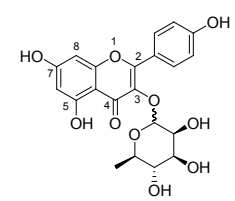

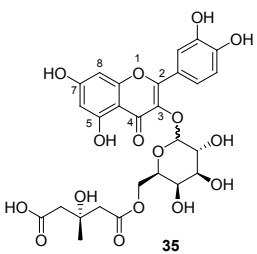

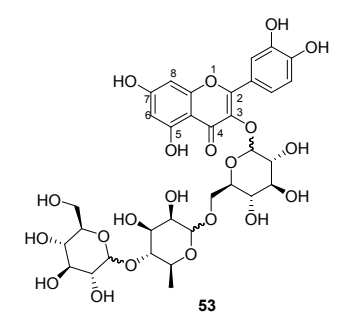

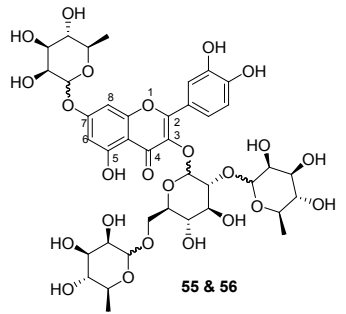

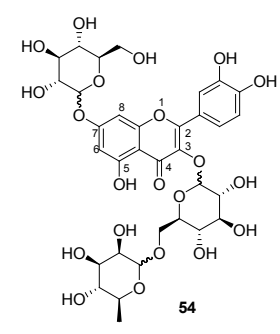

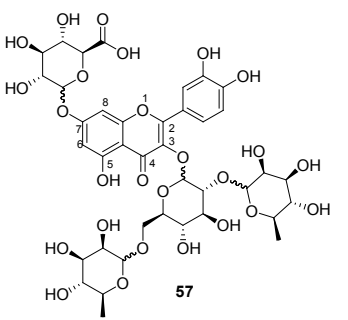

Figure 2: Chemical structures of the phytoconstituents identified in Z. spina-christi. 
patterns representing apigenin 7, 4'-di-O-glucoside 19. Compound 19 produced an $\mathrm{MS}^{2}$ fragment ion at $m / z 431\left([(\mathrm{M}-\mathrm{H})-162]^{-}\right)$by the neutral loss of a glucosyl unit from C-4' (Figure 10S, Supporting information). It produced the $\mathrm{MS}^{3}$ base peak at $\mathrm{m} / z 269$ ([apigenin- $\left.\mathrm{H}\right]^{-}$) by the neutral loss of the second glucosyl unit from C-7 (Table 1). Based on this fragmentation behaviour compound 19 was assigned as apigenin7, 4'-di-O-glucoside.

\section{Characterization of phloretin derivatives (m/z 597)}

Three C-glycosides derivatives of phloretin (compounds 38-40) were detected in the EIC of $Z$. spina-christi leaves extract (Table 1). They produced characteristic $C$-glycosides $\mathrm{MS}^{2}$ fragment ions ([M-H-120 $]^{-}$, $[\mathrm{M}-\mathrm{H}-90]^{-}$and $[\mathrm{M}-\mathrm{H}-30]^{-}$) (Figure 11S, Supporting information). Compound 40 was identified as phloretin 3', 5'-di-C-glucoside. This compound has been previously reported in Ziziphus species [15]. We speculate that compounds 38 and 39 were isomers of compound 40 alternative $C$-glycosylated hexoses. From the literature and our experiments we have found that the glycosides containing galactoside units are more polar than the glycosides containing glucoside units [46]. Based on the above arguments we have tentatively assigned isomer 38 as phloretin $3^{\prime}, 5^{\prime}$-di- $C$-galactoside $\left(\mathrm{t}_{\mathrm{R}} 32.6 \mathrm{~min}\right)$ and isomer 39 as phloretin 3 '- $C$-glucoside 5 '- $C$-galactoside or phloretin 3 '- $C$-galactoside 5 '-C-glucoside $\left(\mathrm{t}_{\mathrm{R}} 36.8 \mathrm{~min}\right)$ (Table 1$)$. To our knowledge, compound 38 was not previously reported in the plant kingdom.

\section{Characterization of diosmetin derivatives $(\mathrm{m} / \mathrm{z} 769)$}

Two peaks, 43 and 44, were detected at $\mathrm{m} / z 769$ in the EIC and showed different retention times ( $t_{\mathrm{R}} 54.4$ and $\left.56.6 \mathrm{~min}\right)$. They showed in their $\mathrm{MS}^{3}$ fragment ions at $\mathrm{m} / z 299$ (base peak), 255 and 284 corresponding to diosmetin aglycone [47], which suggested that, these compounds were diosmetin glycosides (Table 1). Based on the elution order and the data previously reported [48-50] compound 43 was assigned as diosmetin 3'-O-galactoside 7-O-rutinoside and the later eluting isomer 44 as diosmetin 3'-O-glucoside 7-O-rutinoside (Table 1). To the best of our knowledge, compounds 43 and 44 were not reported previously in nature. (Further arguments are provided in the Supporting information).

\section{Characterization isorhamnetin, kaempferol and quercetin derivatives}

Di, tri, tetra and penta glycosylated flavonoids were also detected in Z. spina-christi methanol/water extract. The presence of the aglycone fragment ions at $m / z 315,285$ and 301 in their $\mathrm{MS}^{2}$ and/or MS ${ }^{3}$, and characteristic fragment ions $(\mathrm{m} / z 151$ and 179 for quercetin, $\mathrm{m} / z 151$ for kaempferol and $m / z 300$ for isorhamnetin) [51], in addition to their UV spectra suggested that these compounds were isorhamnetin, kaempferol and quercetin derivatives [52,53].

Compounds 23, 26, 29, 30, 31 and 33 were identified as kaempferol 3-O-(6-O-rhamnosyl-glucoside), quercetin 3-O-(6$O$-rhamnosyl-glucoside) (rutin), quercetin 3-O-arabinoside, quercetin 3-O-rhamnoside, quercetin 3-O-glucoside, and kaempferol 3-O-glucoside, respectively, by the comparison of their UV spectra and retention times with commercial standards.

Compounds 20-22, 24, 25, 27, 28, 32, 34, 37 and 45-57 were tentatively identified as isorhamnetin 3-O-(6-O-rhamnosyl-galactoside), isorhamnetin 3-O-(6-O-rhamnosyl-glucoside), kaempferol 3-O-(6-Orhamnosyl-galactoside), quercetin 7-O-(6-O-rhamnosyl-glucoside), quercetin 3-O-(6-O-rhamnosyl-galactoside), quercetin 3-O-(2-Orhamnosyl-arabinoside), quercetin 3-O-(2-O-rhamnosyl-xyloside), quercetin - $O$-acetyl hexoside, kaempferol 3-O-rhamnoside, quercetin 3-O-[6"-(3-hydroxyl-3-methylglutaryl)- $\beta$-D-galactoside], kaempferol
3-O-[6"-(3-hydroxyl-3-methylglutaryl)- $\beta$-D-galactoside], quercetin 3-O- $\beta$-D-xylosyl- $(1 \rightarrow 2)$ - $\alpha$-L-rhamnoside-4'-O- $\alpha$-L-rhamnoside, kaempferol 3-O-(2, 6-di-O-rhamnosyl-glucoside), quercetin 3-O-(2, 6-di-O-rhamnosyl-glucoside) 7-O-galactoside, quercetin 3-O-(2, 6-di$O$-rhamnosyl-glucoside) 7-O-glucoside, kaempferol 3 -O-rutinoside 7-O-rhamnoside, kaempferol 3-O-(2, 6-di-O-rhamnosyl-glucoside) 7-O-ramnoside, quercetin 3-O-(2, 6-di-O-rhamnosyl-glucoside), quercetin 3-O-rhamnosyl-galactoside 7-O-rhamnoside, quercetin 3-O-rutinoside 7-O-rhamnoside, quercetin 3-O-(rhamnosyl- $(1 \alpha \rightarrow 6)$ $\mathrm{O}$-glucoside) hexoside, quercetin 3 -O-rutinoside 7 -O-glucoside, quercetin 3-O-(2, 6-di-O-rhamnosyl-galactoside) 7-O-rhamnoside, quercetin 3-O-(2, 6-di-O-rhamnosyl-glucoside) 7-O-rhamnoside, and quercetin 3-O-(2, 6-di-O-rhamnosyl-glucoside) 7-O-glucuronide, respectively, as previously reported $[15,26,29,48-50]$ (See also the Supporting information for characterization of these compounds).

Two isomers of flavonoid pentaglycosides (41 and 42) were also detected in the Z. spina-christi methanol/water extract. Both isomers showed a pseudomolecular ion at $m / z 1047\left([\mathrm{M}-\mathrm{H}]^{-}\right)$, and the $\mathrm{MS}^{2}$ spectra showed a base peak at $m / z 901\left([\mathrm{M}-\mathrm{H}-146]^{-}\right)$due to the neutral loss of rhamnoside residue from position 7 of the flavonoid moiety [54] and a high abundance ions $(\geq 60)$ at $755\left([\mathrm{M}-\mathrm{H}-146-146]^{-}\right)$indicating a subsequent neutral loss of another rhamnoside residues from position 7 (Table 1 and Figure 3), this was supported by observing the ion $m / z 755$ as a base peak in $\mathrm{MS}^{3}$ spectra. Their MS ${ }^{4}(755 \rightarrow 300 / 301)$ [neutral loss of triglyosides $(454 \mathrm{u})$ moieties] were reminiscent to that of quercetin 3-O(2, 6-di-O-rhamnosyl-glucoside) (50) (Table 1). These data suggested that these isomers were derivatives of compound 50 with an additional two $O$-rhamnoside substituent at position 7. Accordingly, isomer 42 was tentatively identified as quercetin 3-O-(2, 6-di-O-rhamnosylglucoside) 7-di-O-rhamnoside, and the earlier-eluted isomer (41) as quercetin 3-O-(2, 6-di-O-rhamnosyl-galactoside) 7-di- $O$-rhamnoside. To the best of our knowledge compounds 41 and 42 were not reported previously in nature.

\section{Characterization of the isolated constituents}

In our present work, preparative HPLC was used after the purification on $\mathrm{C}_{18}$ solid phase extraction columns to isolate four known compounds from the methanol/water $(7: 3 \mathrm{v} / \mathrm{v})$ extract of $Z$. spina-christi leaves. The structures of the isolated compounds were elucidated by HRMS (Table S1 in the Supporting information), tandem MS (Table 1 and Figure 4), UV chromatograms (Figure 1), and authentic standards. (See also Figures $18 \mathrm{~S}$ and $19 \mathrm{~S}$ in the Supporting information). Chromatographic resolution and MS data were considered for isolated compounds showing a poor NMR spectral resolution. With these agreements compound $26(25.0 \mathrm{mg})$ was identified as quercetin 3-O-(6$O$-rhamnosyl-glucoside) (rutin), compound $27(2.6 \mathrm{mg})$ as quercetin 3-O-(2-O-rhamnosyl-arabinoside), compound $39(9.0 \mathrm{mg})$ as phloretin $3^{\prime}, 5^{\prime}$ di-C-glycoside and compound $50(2.5 \mathrm{mg})$ as quercetin $3-O-(2,6-$ di-O-rhamnosyl-glucoside) (Figure 2). Compounds 26, 27 and 39 have been previously isolated from the fruits of Ziziphus species [15]. The UV spectra, MS fragmentation and the retention time of compound 50 were compared with the compound $(m / z 755)$ reported in the leaves of Catharanthus roseus and found that they are identical [55]. The purity of these compounds was determined as in our previous study [29] by total ion chromatograms in negative ion mode and UV chromatograms at 280 and $320 \mathrm{~nm}$. The purity of the compounds were $86 \%$ for compound $26,75 \%$ for compound $27,56 \%$ for compound 39 and $>98 \%$ for compound 50 .

\section{Antibacterial activity}

The antibacterial activities of the plant extracts analysed in this 


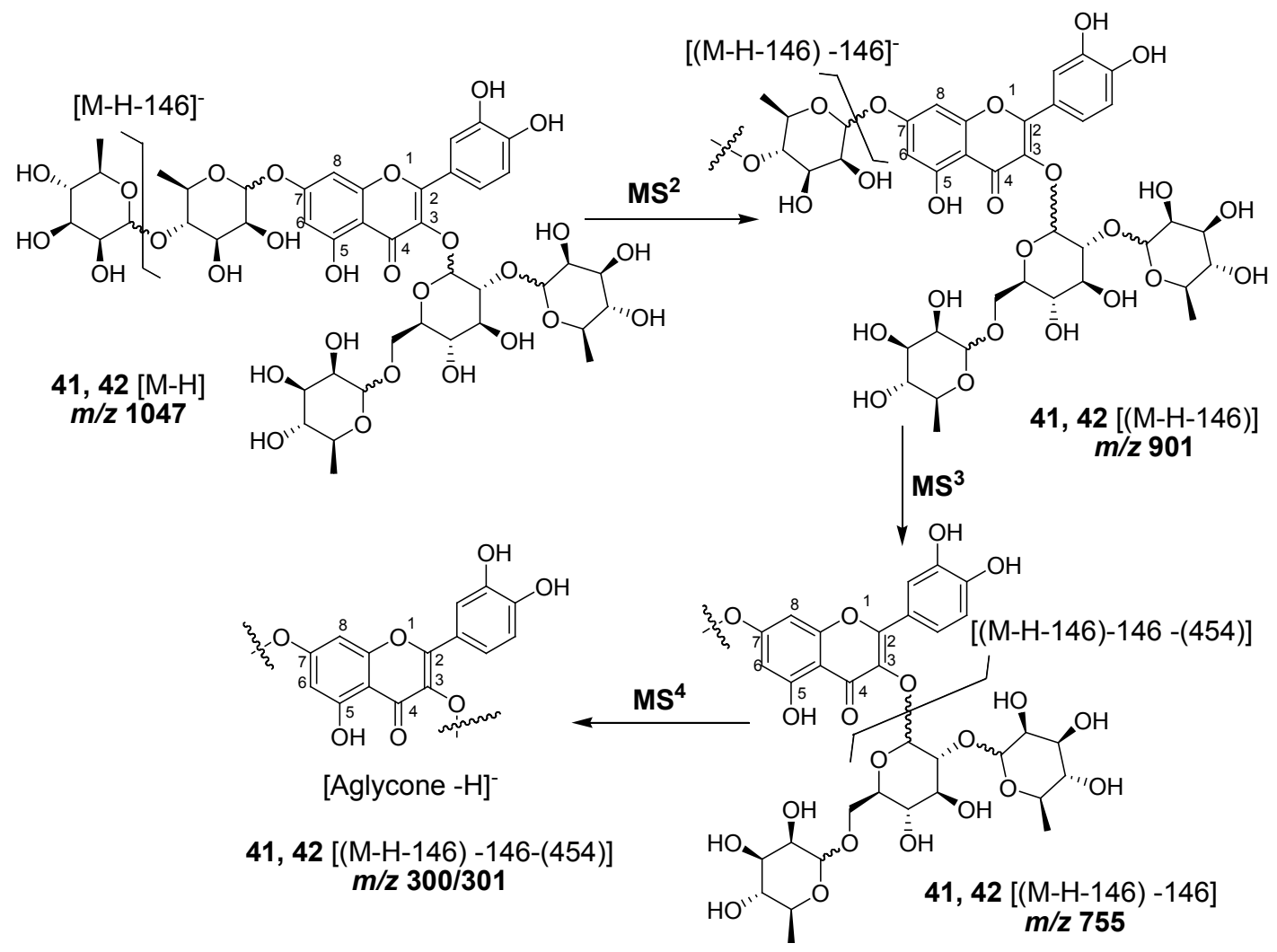

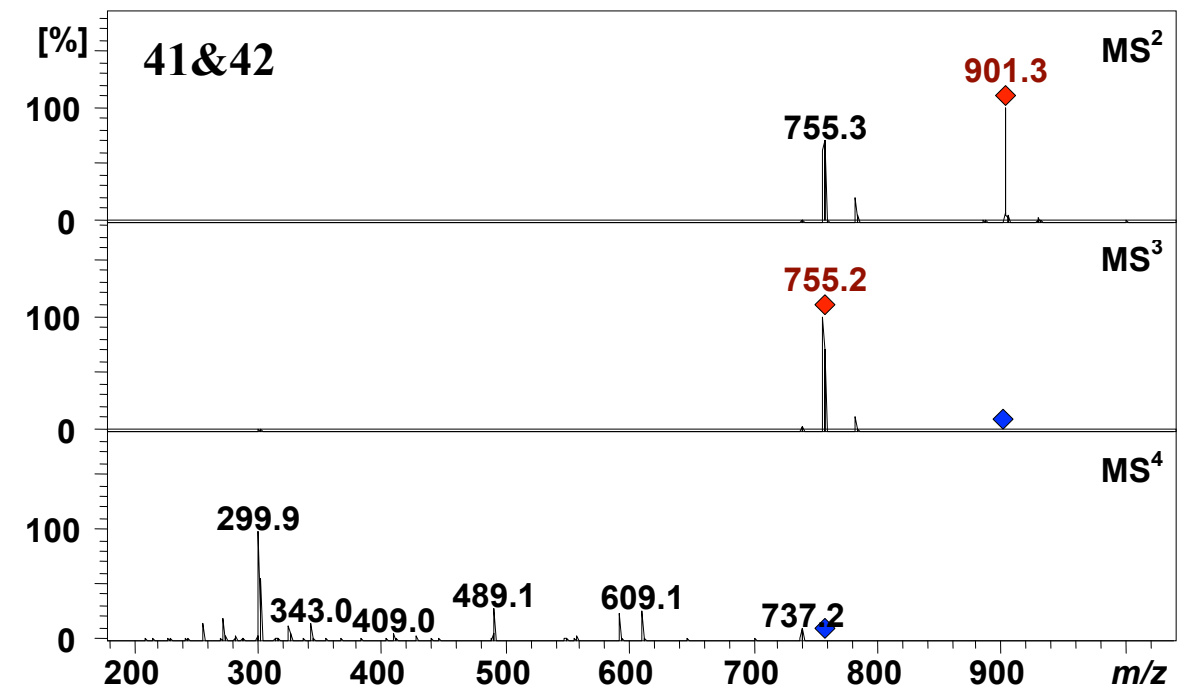

Figure 3: Proposed fragmentation pathway and $\mathrm{MS}^{4}$ spectra of quercetin 3-O-(2, 6-di-O-rhamnosyl-galactoside) 7-di-O-rhamnoside 41 and quercetin 3-O-(2, 6-di-O-rhamnosyl-glucoside) 7-di-O-rhamnoside 42 of precursor ion at $\mathrm{m} / \mathrm{z} 1047$ in negative ion mode.

study were evaluated for their efficacies against Gram-positive and Gram-negative bacteria using the agar diffusion method. As suitable model organisms B. subtilis S168, B. aquimaris MB-2011, and C. michiganensis GSPB 390 were chosen for Gram-positive and E. coli DH5a, E. amylovora 1189, and P. syringae pv tomato DC300 for Gramnegative. The results of the antibacterial activity tests of aqueous and methanol extracts of the leaves of Z. spina-christi are given in Table 2 and Figure 5. Interestingly, the plant extracts tested herein showed antibacterial activity only against the Gram-positive bacterium $B$. aquimaris MB2011 and the Gram-negative representative P. syringae pv tomato DC300 while the other bacterial strains were not affected at all. These results are in contrast to those of others [56] who found that ethanol and methanol extracts of $Z$. spina-christi leaves inhibited the growth of a variety of Gram-negative bacterial strains Salmonella typhi, Proteus mirabilis, Shigella dysenteriae, E. coli, Klebsiella pneumoniae, Brucella melitensis, Bordetella bronshiseptica and Pseudomonas aeruginosa. Previously, Moghadam et al. reported antibacterial activity of ethanolic extracts of the leaves of $Z$. spina-christi against a Grampositive, Methicillin-resistance Staphylococcus aureus strain [9]. 
Citation: Elsadig Karar MG, Quiet L, Rezk A, Jaiswal R, Rehders M, et al. (2016) Phenolic Profile and In Vitro Assessment of Cytotoxicity and Antibacterial Activity of Ziziphus spina-christi Leaf Extracts. Med chem (Los Angeles) 6: 143-156. doi:10.4172/2161-0444.1000339
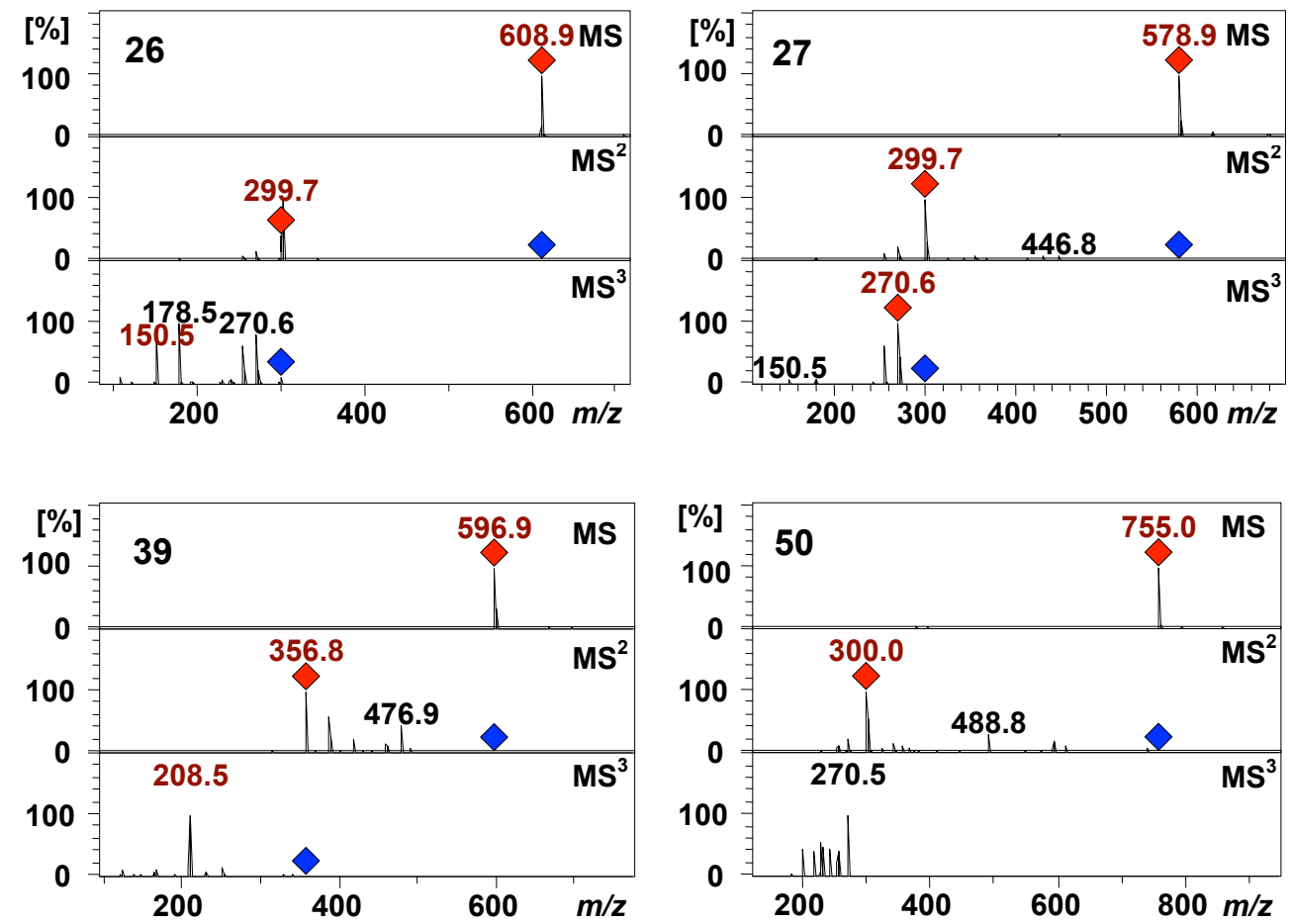

Figure 4: Tandem MS spectra of the isolated constituents in negative ion mode.

\begin{tabular}{|c|c|c|c|c|c|}
\hline \multirow[t]{3}{*}{ Bacterial strains } & \multicolumn{5}{|c|}{ Inhibition zone diameters (mm) } \\
\hline & \multicolumn{2}{|c|}{ Extracts prepared in } & \multicolumn{3}{|c|}{ Antibiotic } \\
\hline & Water & $\mathrm{MeOH}$ & Kanamycin & Ampicillin & Streptomycin \\
\hline \multicolumn{6}{|l|}{ Gram-positive } \\
\hline B. subtilis (S168) & $0.0 \pm 0.0$ & $0.0 \pm 0.0$ & $20.0 \pm 0.0$ & NA & NA \\
\hline B. aquimaris (MB2011) & $16.0 \pm 1.0$ & $26.0 \pm 2.0$ & $19.7 \pm 0.6$ & NA & NA \\
\hline C. michiganensis (GSPB 390) & $0.0 \pm 0.0$ & $0.0 \pm 0.0$ & NA & NA & $10.3 \pm 0.6$ \\
\hline \multicolumn{6}{|l|}{ Gram-negative } \\
\hline E. coli $(\mathrm{DH} 5 \alpha)$ & $0.0 \pm 0.0$ & $0.0 \pm 0.0$ & NA & $16.3 \pm 0.6$ & NA \\
\hline E. amylovora (1189) & $0.0 \pm 0.0$ & $0.0 \pm 0.0$ & NA & $13.3 \pm 0.6$ & NA \\
\hline P. syringae pv tomato (DC300) & $0.0 \pm 0.0$ & $18.3 \pm 0.5$ & NA & NA & $23.3 \pm 1.5$ \\
\hline
\end{tabular}

NA = Not Applicable

Table 2: Antibacterial activities of $Z$. spina-christi leaf extracts and the standard antibiotics as assessed by the agar diffusion method.

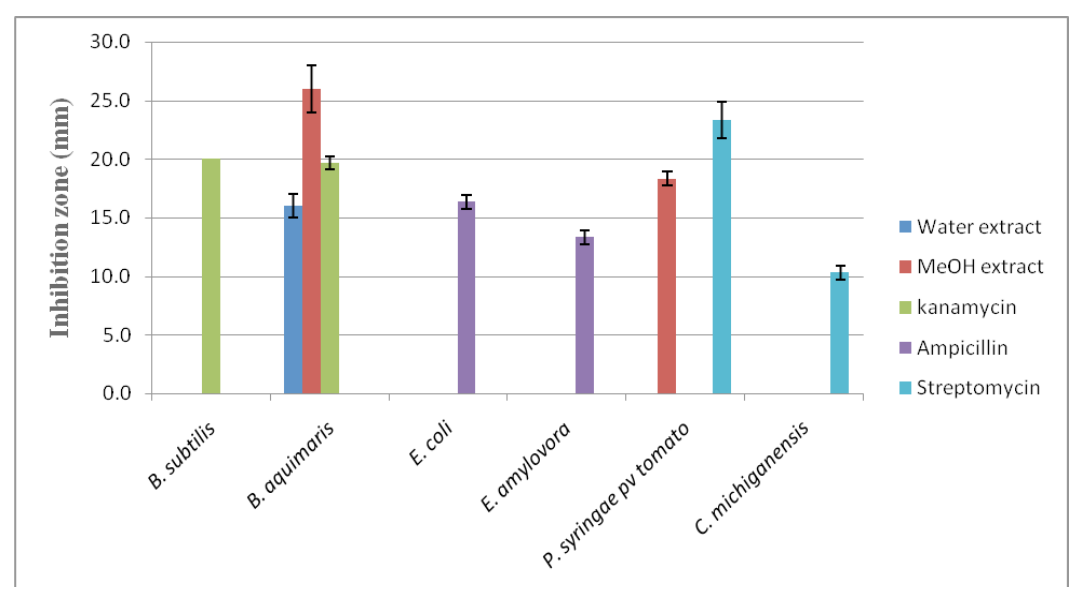

Figure 5: Inhibition zones as means \pm SD generated by extracts prepared from Z. spina-christi leaves and the standard antibiotics against bacterial species tested. 
In line with our findings, it has been shown before that many plant metabolites including flavonoids, phenols, tannins, and alkaloids exhibit moderate antimicrobial activity [57]. Our data propose that antibacterial activities might be associated with the phenolic compounds from Z. spina-christi leaves, which were identified in the aqueous and methanol extracts. Our data furthermore indicated that methanol extracts appeared to be more effective in antibacterial activity against Gram-negative bacteria as compared to the aqueous extracts of the leaves from $Z$. spina-christi suggesting that the bioactive metabolites of $Z$. spina-christi were rather hydrophobic than hydrophilic. However, B. aquimaris MB-2011 was similarly affected by the aqueous and the methanol extracts suggesting that it is either affected by at least two differently extractable compounds or that it is susceptible to a compound different from that effective against the Gram-negative strain $P$. syringae. Thus, if traditional use of the leaves of $Z$. spina-christi involves the preparation of a pre-extract in alcoholic solvents, their effectiveness may be more pronounced than simply brewing the leaves in water [3].

\section{Cytotoxic activity}

In order to use Z. spina-christi extract as a potential antibiotic and to enable safe use as topical treatment of skin or as systemic drug taken up by oral administration routes, cytotoxicity against the exposed cells and tissues must be analyzed in order to exclude potential negative, offtarget effects during treatment. Here, we have used human keratinocyte $(\mathrm{HaCaT})$ and rat intestine epithelial (IEC-6) cell lines in culture to assess potential cytotoxicity exerted by leaf extracts from $Z$. spina-christi in vitro. For all experiments, the cells were first grown to confluence with the aim to simulate the effect of the extracts on cell monolayers which are considered representative of intact epithelia of the respective tissue, i.e., epidermis of the skin and mucosa of the gastro-intestinal tract lining.

Cytotoxicity was estimated by using the 3-(4, 5-dimethylthiazol2-yl)-2, 5-diphenyltetrazolium bromide (MTT) assay, which is a routine test evaluating cell viability and proliferative activity by means of cellular enzyme activity. The MTT is converted by mitochondrial NADH-dependent dehydrogenase into an insoluble dye made of dark blue formazan crystals [58]. MTT assays are suited to determine the activity of mitochondrial enzymes reducing the yellow tetrazole MTT to purple formazan in living cells, only [59]. Therefore MTT conversion is proportional to both, cell viability and proliferation rates of treated cell cultures.

The MTT assay has been applied by us and others before. In comparison with other cytotoxicity assays such as the neutral red assay or quantification of LDH latency, the MTT assay was suitable but less sensitive than the former when $\mathrm{CdCl}_{2}$ effects onto hepatoma cell lines were determined [60]. In another study, the above mentioned three different assays were compared and additionally ATP contents of the cultured cells were determined [61]. While both, the MTT and the $\mathrm{LDH}$ assay were somewhat non-suitable because of their sensitivity to inhibitory agents contained in the compound mixtures tested, it was also pointed out that the choice of assay depends on the pathway of death that is potentially initiated by the agents applied.

Here, we used the MTT assay to recapitulate the well-known cytotoxicity of DMSO by incubating cultures of HaCaT keratinocytes and IEC6 intestine epithelial cells with different concentrations thereof (Figure 20S, Supporting information). The results demonstrate that indeed DMSO is cytotoxic if applied at concentrations above $1 \%$, because MTT conversion declines in a concentration dependent
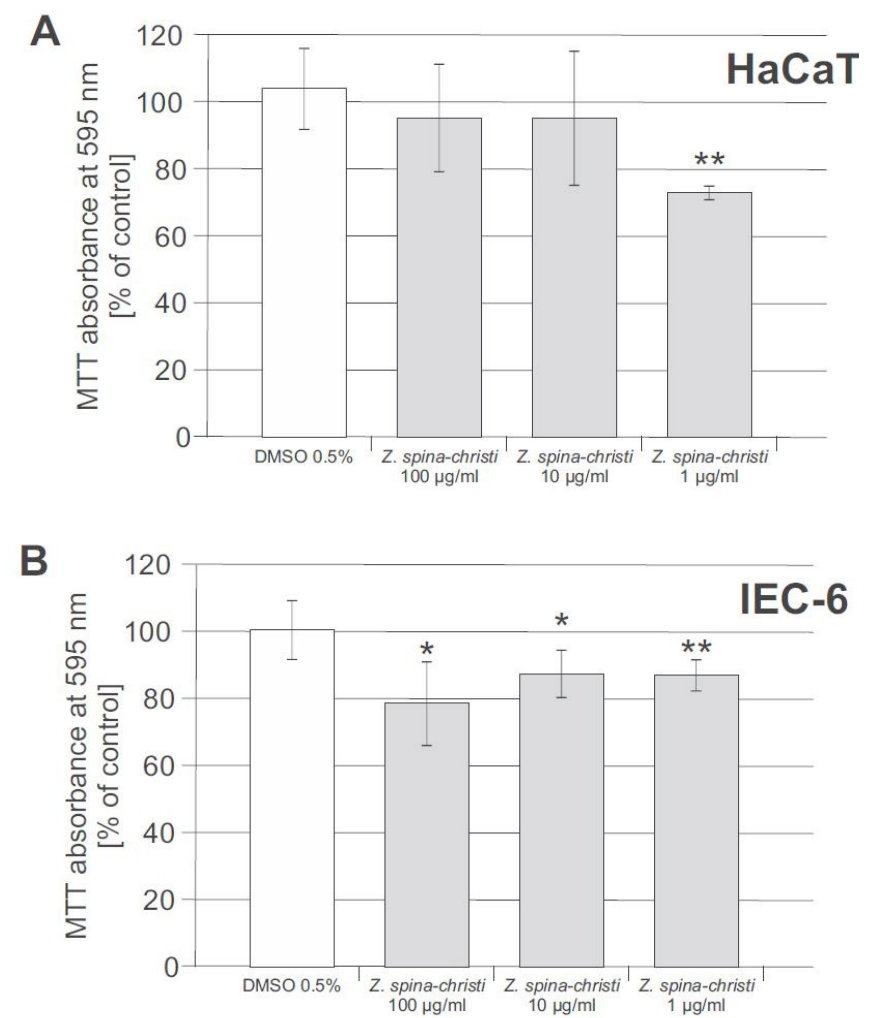

Figure 6: MTT conversion by HaCaT keratinocytes (A) and IEC-6 small intestine epithelial cells (B) upon incubation with 1,10 or $100 \mu \mathrm{g} / \mathrm{ml} Z$. spinachristi extracts for $24 \mathrm{~h}(\mathrm{n}=3)$. Extent of MTT conversion was quantified spectrophotometrically and values are given as means \pm standard deviations. Cells treated with $0.5 \%$ DMSO, only, were used as controls and set to $100 \%$.

manner. However, the data also support the notion that concentrations of up to $0.5 \%$ DMSO are non-cytotoxic, and can be used to as solvent of lipophilic compounds.

We have used this assay before to determine cell viability upon exposure of fibroblasts, keratinocytes, and intestine epithelial cells to other stressors in addition to testing for the safe use of e.g., potential drug delivery tools $[33,34,62]$. The conversion of MTT by HaCaT keratinocytes (Figure 6A) is not influenced upon exposure to $100 \mu \mathrm{g}$ or $10 \mu \mathrm{g} / \mathrm{ml} \mathrm{Z}$. spina-christi methanol/water $(7: 3 \mathrm{v} / \mathrm{v})$ extract. However, upon treatment with $1 \mu \mathrm{g} / \mathrm{ml} \mathrm{Z}$. spina-christi conversion of MTT was decreased in HaCaT cell cultures. On the other hand the MTT conversion by small intestine epithelial IEC-6 cells (Figure 6B) increased with decreasing concentration although this was not statistically significantly different from controls. For the lower concentrations of 10 $\mu \mathrm{g}$ and $1 \mu \mathrm{g} / \mathrm{ml}$ the values of conversion were approximately even. We conclude that cytotoxicity towards IEC-6 cell cultures increased with increasing concentration of Z. spina-christi, and it was safe to apply extracts at a final concentration of $1 \mu \mathrm{g} / \mathrm{ml}$ to intestine epithelial cell monolayers. In contrast, for $\mathrm{HaCaT}$ cells the exposure to $1 \mu \mathrm{g} / \mathrm{ml} Z$. spina-christi extracts, only exerted cytotoxic effects that were detectable by a significant reduction of the MTT-conversion to approx. $75 \%$ in comparison to DMSO-treated controls.

In addition, MTT- and LDH-assays are usually backed up by additional tests that involve determination of cellular integrity as we have shown in a study in which the MTT assay was employed to test for cytotoxicity of dust samples applied to keratinocytes and fibroblasts [34]. Results achieved by the MTT assay were comparable with culture 
impedance measurements. In addition, phalloidin staining of the actin cytoskeleton and propidium iodide staining of the nuclei of cells with ruptured plasma membrane were performed in our previous work, again, supporting the results of the MTT-assay with regard to structural and metabolic integrity of the cells, respectively. In another study, the MTT assay was used by us to determine $\mathrm{IC}_{50}$ values of cucurbit [7] uril which were backed up by imaging studies and supported by in vivo toxicity studies revealing highly congruent data [62]. Therefore, we believe that the MTT assay, especially when used in combination with additional measures of cytotoxicity, is a suitable method to conclude on cytotoxic effects of diverse samples such as dust simulants, drug delivery agents, or plant extracts as applied in this study.

Consequently, here we performed propidium iodide (PI) staining for both, HaCaT keratinocytes and IEC-6 intestine epithelial cells to determine the extent of potential plasma membrane rupture and the number of necrotic cells per viable cells when the cultures were treated with extracts of $Z$. spina-christi. This assay is based on the fact that PI intercalates with DNA, thereby visualizing nuclear DNA when PI passes ruptured membrane lipid bilayers. Therefore PI may stain for necrotic cells in which the lipid bilayer integrity of the plasma membrane is compromised [34]. With increasing concentration of Z. spina-christi extracts, the number of necrotic or ruptured cells increased for the exposed HaCaT cultures (Figure 7A). For the IEC-6 cell cultures (Figure 7B), the number of ruptured cells decreased upon exposure to leaf extract of $Z$. spina-christi. However, the numbers of ruptured cells in $\mathrm{HaCaT}$ or IEC6 cell cultures treated with Z. spina-christi leaf extracts remained moderate as they were determined to not even reach one percent upon $24 \mathrm{~h}$ of exposure.

As an additional and sensitive measure of cellular integrity, the actin cytoskeleton was analyzed using a Phalloidin Draq5 ${ }^{\text {Tx }}$ staining.

A

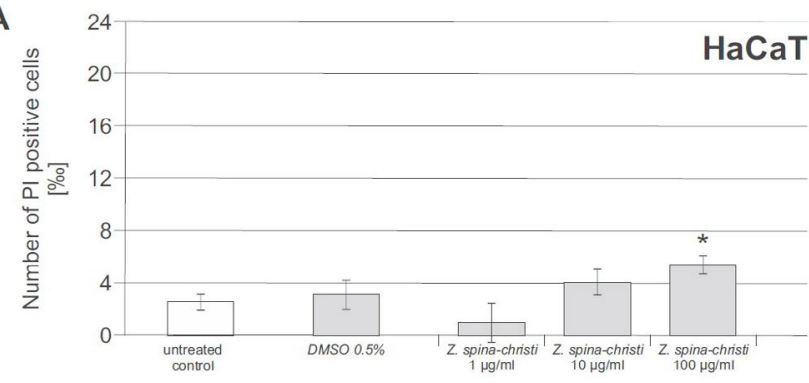

B

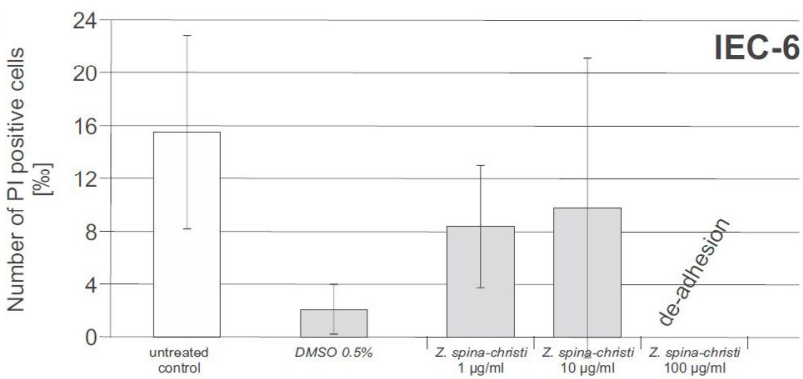

Figure 7: Plasma membrane integrity and extent of necrotic cell death exerted after 24 hours of exposure of $\mathrm{HaCaT}$ keratinocytes (A) or IEC-6 small intestine epithelial cells (B) to leaf extracts from Z. spina christi. The acquisition of $\mathrm{PI}$ indicates ruptured plasma membranes due to necrotic cell death. Fluorescence intensities of $\mathrm{PI}$ in three arbitrary chosen regions were determined by CellProfiler ${ }^{\mathrm{TM}}$ software to quantify the fluorescence of dead cells over that of all cells, which was determined by Draq5 $5^{\mathrm{TM}}$ counter-staining of nuclear DNA. Values are given as permille and expressed as means \pm standard deviations.

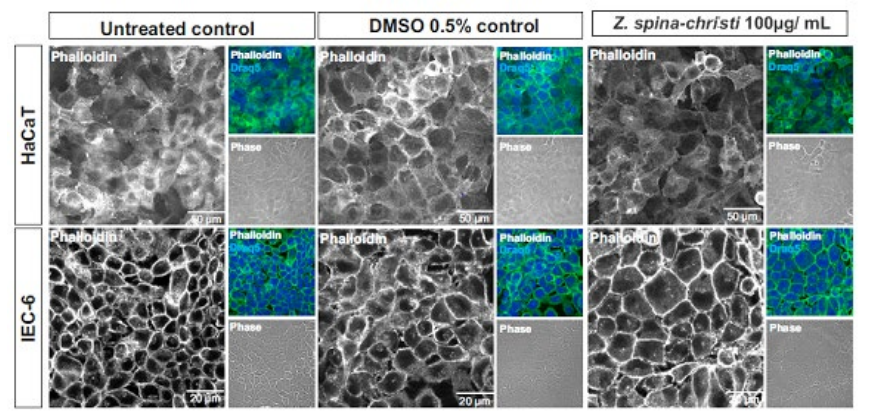

Figure 8: Phalloidin staining of the filamentous actin system of $\mathrm{HaCaT}$ keratinocytes and IEC- 6 small intestine epithelial cells after exposure to 100 $\mu \mathrm{g} / \mathrm{ml}$ leaf extracts of $Z$. spina-christi for $24 \mathrm{~h}$. Single channel fluorescence, phase contrast and merged micrographs taken with a confocal laser scanning microscope of formaldehyde-fixed cells after staining of actin filaments with FITC-phalloidin, and Draq5 ${ }^{\mathrm{TM}}$ counter-staining of nuclear DNA. Bars represent $20 \mu \mathrm{m}$ and $50 \mu \mathrm{m}$, respectively.

Upon treatment with $100 \mu \mathrm{g} / \mathrm{ml}$ leaf extracts of Z. spina-christi, HaCaT cells (Figure 8 ) showed a slightly altered actin filamentous system, and some cells detached from their neighbors indicating loosening of cellcell contacts. For the IEC-6 cell cultures (Figure 8), the cortical F-actin system remained intact upon treatment with different concentrations of the extracts prepared from Z. spina-christi.

In summary, mild cytotoxic effects can be observed on both, $\mathrm{HaCaT}$ and IEC- 6 cell cultures upon a 24-h treatment with leaf extracts of $Z$. spina-christi. IEC-6 cells were less sensitive compared to HaCaT cells, suggesting $Z$. spina-christi extracts might be potentially suitable for topical applications.

\section{Conclusions}

Z. spina-christi is a medicinal and edible plant growing wild in Sudan and used for a long time in traditional medicine for the treatment of different diseases caused by infection. In the present work we report that $Z$. spina-christi leaves are a rich source of phenolic compounds including chlorogenic acids, proanthocyanidins, simple and highly glycosylated flavonoids. Fifty-seven components were tentatively detected and characterized on the basis of reported analytical data from three to four pieces of independent methods including retention time, UV/Vis spectroscopy, high resolution mass spectrometry (HR$\left.\mathrm{MS}^{\mathrm{n}}\right)$ and tandem mass spectrometry $\left(\mathrm{MS}^{\mathrm{n}}\right)$. To our knowledge 45 of them were not reported previously in $Z$. spina-christi and five for the first time in nature. Besides, four bioactive known compounds were isolated. Next, four known phenolic compounds were isolated and characterized by means of preparative-HPLC and other spectroscopic techniques. We investigated the antimicrobial activities of aqueous and methanol extracts of $Z$. spina-christi leaves. Methanol extracts were more effective in inhibiting bacterial growth than the aqueous extracts, which is most likely attributed to the solubility and hydrophobicity of the antibacterial plant compounds. Remarkably, the methanol extract of $Z$. spina-christi leaves revealed an antibacterial activity against both, a Gram-positive and a Gram-negative tester organism while the aqueous extracts also showed milder activity against the Gram-positive representative, B. aquimaris. We conclude that further studies are needed with the goal to purify the methanol extracts in order to isolate and identify the specific compound/s that exert/s antimicrobial effects, in particular, against human pathogens. Furthermore, the cytotoxicity of $Z$. spina-christi leaf extracts was tested with a range of different cell biological and biochemical means. The herein established cytotoxicity assessment routine revealed that short-term application of $Z$. spina- 
Citation: Elsadig Karar MG, Quiet L, Rezk A, Jaiswal R, Rehders M, et al. (2016) Phenolic Profile and In Vitro Assessment of Cytotoxicity and Antibacterial Activity of Ziziphus spina-christi Leaf Extracts. Med chem (Los Angeles) 6: 143-156. doi:10.4172/2161-0444.1000339

christi leaf extracts barely exhibited any cytotoxic effects on human skin keratinocytes $(\mathrm{HaCaT})$ or rat small intestine epithelial cells (IEC-6). This was deduced from analyses of plasma membrane integrity as well as the intracellular cytoskeletal architecture, which remained largely unaffected upon short-term treatment with the leaf extracts. Moreover, activities of the mitochondrial reductase were not affected by $Z$. spinachristi extract incubation of IEC6 cells or HaCaT keratinocyte cultures. In conclusion, the above outlined assessment routine has proved suitable to test for possible cytotoxic effects of crude plant extracts in a straight-forward approach, thereby allowing to conclude on the integrity of biological membranes, structural elements such as the actin cytoskeleton, and on the metabolic and proliferative activities of HaCaT keratinocyte and IEC6 intestine epithelial cells.

\section{Additional File}

High resolution MS data, full assignment arguments, proposed fragmentation pathways and $\mathrm{MS}^{\mathrm{n}}$ spectra of some compounds are provided in the Supporting information.

\section{Acknowledgements}

This work was supported by Jacobs University Bremen and Deutscher Akademischer Austausch Dienst (DAAD). The authors would like to thank Anja Müller for excellent technical support. We are grateful to Dr. Hayder Abdelgader, Medicinal and Aromatic Plants Research Institute (MAPRI), Khartoum, Sudan, for providing the plant materials.

\section{References}

1. Lucas A (1931) Cedar-Tree products employed in mummification. J Egyp Archaeol 17: 13-21.

2. Dafni A, Levy S, Lev E (2005) The ethnobotany of Christ's Thorn Jujube (Ziziphus spina-christi) in Israel. J Ethnobiol Ethnomed 1: 8

3. Saied AS, Gebauer J, Hammer K, Buerkert A (2008) Ziziphus spina-christi (L.) Willd: A multipurpose fruit tree. Genet Resour Crop Evol 55: 929-937.

4. Kirtikar KR, Basu B (1984) Indian Medicinal Plants. 2nd edn. Delhi: Periodical Expert Book Agency.

5. Han BH, Park MH (1986) Folk Medicine: The Art and the Science. 6th edn. Washington, DC: The American Chemical Society.

6. El-Kamali HH, El-Khalifa KF (1999) Folk medicinal plants of riverside forests of the Southern Blue Nile district, Sudan. Fitoterapia 70: 493-497.

7. Michel CG, Nesseem DI, Ismail MF (2011) Anti-diabetic activity and stability study of the formulated leaf extract of Zizyphus spina-christi (L.) Willd with the influence of seasonal variation. J Ethnopharmacol 133: 53-62.

8. Glombitza KW, Mahran GH, Mirhom YW, Michel KG, Motawi TK (1994) Hypoglycemic and antihyperglycemic effects of Zizyphus spina-christi in rats Planta Med 60: 244-247.

9. Moghadam MS, Maleki S, Darabpour E, Motamedi H, Nejad SMS (2010) Antibacterial activity of eight Iranian plant extracts against methicillin and cefixime restistant Staphylococcous aureus strains. Asian J Trop Med 3: $262-$ 262.

10. Hadizadeh I, Peivastegan B, Kolahi M (2009) Antifungal activity of nettle (Urtica dioica L.), colocynth (Citrullus colocynthis L. Schrad), oleander (Nerium oleander L.) and konar (Ziziphus spina-christi L.) extracts on plants pathogenic fungi. Pakistan J Biol Sci 12: 58-63.

11. Adzu B, Amos S, Wambebe C, Gamaniel K (2001) Antinociceptive activity of Zizyphus spina-christi root bark extract. Fitoterapia 72: 344-350.

12. Ruiz J (2003) Mechanisms of resistance to quinolones: target alterations, decreased accumulation and DNA gyrase protection. J Antimicrob Chemother 51: 1109-1117.

13. Li XZ, Nikaido H (2009) Efflux-mediated drug resistance in bacteria: an update. Drugs 69: 1555-1623.

14. Ben HA, Hamdi N (2012) Phytochemical composition and antimicrobial activities of the essential oils and organic extracts from Pelargonium graveolens growing in Tunisia. Lipids Health Dis 11: 167
15. Pawlowska AM, Camangi F, Bader A, Braca A (2008) Flavonoids of Zizyphus jujuba L. and Zizyphus spina-christi (L.) Willd (Rhamnaceae) fruits. Food Chem 112: 858-862.

16. Randhir R, Lin YT, Shetty K (2004) Phenolics, their antioxidant and antimicrobial activity in dark germinated fenugreek sprouts in response to peptide and phytochemical elicitors. Asia Pac J Clin Nutr 13: 295-307.

17. Bors W, Saran M (1987) Radical scavenging by flavonoid antioxidants. Free Radic Res Commun 2: 289-294.

18. Cowan MM (1999) Plant products as antimicrobial agents. Clin Microbiol Rev 12: $564-582$.

19. Moroney MA, Alcaraz MJ, Forder RA, Carey F, Hoult JR (1988) Selectivity of neutrophil 5-lipoxygenase and cyclo-oxygenase inhibition by an antiinflammatory flavonoid glycoside and related aglycone flavonoids. J Pharm Pharmacol 40: 787-792.

20. Van Wauwe J, Goossens J (1983) Effects of antioxidants on cyclooxygenase and lipoxygenase activities in intact human platelets: comparison with indomethacin and ETYA. Prostaglandins 26: 725-730.

21. Amellal M, Bronner C, Briancon F, Haag M, Anton R, et al. (1985) Inhibition of mast cell histamine release by flavonoids and biflavonoids. Planta Med 51: 16-20.

22. Kris-Etherton PM, Hecker KD, Bonanome A, Coval SM, Binkoski AE, et al. (2002) Bioactive compounds in foods: their role in the prevention of cardiovascular disease and cancer. Am J Med 113 Suppl 9B: 71S-88S.

23. Hertog MG, Feskens EJ, Hollman PC, Katan MB, Kromhout D (1993) Dietary antioxidant flavonoids and risk of coronary heart disease: the Zutphen Elderly Study. Lancet 342: 1007-1011.

24. Shahat AA, Pieters L, Apers S, Nazeif NM, Abdel-Azim NS, et al. (2001) Chemical and biological investigations on Zizyphus spina-christi L. Phytother Res 15: 593-597.

25. Tripathi M, Pandey MB, Jha RN, Pandey VB, Tripathi PN, et al. (2001) Cyclopeptide alkaloids from Zizyphus jujuba. Fitoterapia 72: 507-510.

26. Guo S, Duan JA, Tang Y, Qian Y, Zhao J, et al. (2011) Simultaneous qualitative and quantitative analysis of triterpenic acids, saponins and flavonoids in the leaves of two Ziziphus species by HPLC-PDA-MS/ELSD. J Pharm Biomed Anal 56: $264-270$.

27. Clifford MN, Kirkpatrick J, Kuhnert N, Roozendaal H, Salgado PR (2008) LC MSn analysis of the cis isomers of chlorogenic acids. Food Chem 106: 379-385.

28. Jaiswal R, Jayasinghe L, Kuhnert N (2012) Identification and characterization of proanthocyanidins of 16 members of the Rhododendron genus (Ericaceae) by tandem LC-MS. J Mass Spectrom 47: 502-515.

29. Karar MGE, Pletzer D, Jaiswal R, Weingart H, Kuhnert N (2014) Identification, characterization, isolation and activity against Escherichia coli of quince (Cydonia oblonga) fruit polyphenols. Food Res Int 65: 121-129.

30. Nathan P, Law EJ, Murphy DF, MacMillan BG (1978) A laboratory method for selection of topical antimicrobial agents to treat infected burn wounds. Burns 4: 177-187.

31. Buth H, Luigi Buttigieg P, Ostafe R, Rehders M, Dannenmann SR, et al. (2007) Cathepsin $B$ is essential for regeneration of scratch-wounded normal human epidermal keratinocytes. Eur J Cell Biol 86: 747-761.

32. Buth $H$, Wolters B, Hartwig B, Meier-Bornheim R, Veith $H$, et al. (2004) HaCaT keratinocytes secrete lysosomal cysteine proteinases during migration. Eur $\mathrm{J}$ Cell Biol 83: 781-795.

33. Mayer K, Vreemann A, Qu H, Brix K (2009) Release of endo-lysosomal cathepsins $B, D$, and $L$ from IEC6 cells in a cell culture model mimicking intestinal manipulation. Biol Chem 390: 471-480.

34. Rehders M, Grosshaeuser BB, Smarandache A, Sadhukhan A, Mirastschijsk $U$, et al. (2011) Effects of lunar and mars dust simulants on HaCaT keratinocytes and CHO-K1 fibroblasts. Adv Space Res 47: 1200-1213.

35. Carpenter AE, Jones TR, Lamprecht MR, Clarke C, Kang IH, et al. (2006) CellProfiler: image analysis software for identifying and quantifying cell phenotypes. Genome Biol 7: R100.

36. Abad-GarcÃa B, Garmon-Lobato S, Berrueta LA, Gallo B, Vicente F (2009) Practical guidelines for characterization of O-diglycosyl flavonoid isomers by triple quadrupole MS and their applications for identification of some fruit juices flavonoids. J Mass Spectrom 44: 1017-1025 
Citation: Elsadig Karar MG, Quiet L, Rezk A, Jaiswal R, Rehders M, et al. (2016) Phenolic Profile and In Vitro Assessment of Cytotoxicity and Antibacterial Activity of Ziziphus spina-christi Leaf Extracts. Med chem (Los Angeles) 6: 143-156. doi:10.4172/2161-0444.1000339

37. Abad-Garcia B, Berrueta LA, Garmon-Lobato S, Gallo B, Vicente F (2009) A general analytical strategy for the characterization of phenolic compounds in fruit juices by high-performance liquid chromatography with diode array detection coupled to electrospray ionization and triple quadrupole mass spectrometry. J Chromatogr A 1216: 5398-5415.

38. Gu L, Kelm MA, Hammerstone JF, Zhang Z, Beecher G, et al. (2003) Liquid chromatographic/electrospray ionization mass spectrometric studies of proanthocyanidins in foods. J Mass Spectrom 38: 1272-1280.

39. Kuhnert N (2010) Unraveling the structure of the black tea thearubigins. Arch Biochem Biophys 501: 37-51.

40. Clifford MN, Johnston KL, Knight S, Kuhnert N (2003) Hierarchical scheme for LC-MSn identification of chlorogenic acids. J Agric Food Chem 51: 2900-2911.

41. Jaiswal R, Kuhnert N (2010) Hierarchical scheme for liquid chromatography/ multi-stage spectrometric identification of 3,4,5-triacyl chlorogenic acids in green Robusta coffee beans. Rapid Commun Mass Sp 24: 2283-2294.

42. Jaiswal R, Kuhnert $N$ (2011) Identification and characterization of five new classes of chlorogenic acids in burdock (Arctium lappa L.) roots by liquid chromatography/tandem mass spectrometry. Food Funct 2: 63-71.

43. Kuhnert N, Jaiswal R, Matei MF, Sovdat T, Deshpande S (2010) How to distinguish between feruloyl quinic acids and isoferuloyl quinic acids by liquid chromatography/tandem mass spectrometry. Rapid Commun Mass Sp 24: 1575-1582.

44. Lin L, Harnly JM (2010) Identification of the phenolic components of chrysanthemum flower (Chrysanthemum morifolium Ramat). Food Chem 120: 319-326.

45. Jaiswal R, Kuhnert N (2014) Identification and characterization of the phenolic glycosides of Lagenaria siceraria Stand. (bottle gourd) fruit by liquid chromatography-tandem mass spectrometry. J Agric Food Chem 62: 12611271.

46. Kite GC, Veitch NC (2009) Assigning glucose or galactose as the primary glycosidic sugar in 3-O-mono-, di- and triglycosides of kaempferol using negative ion electrospray and serial mass spectrometry. Rapid Commun Mass Sp 23: 3125-3132.

47. Gattuso G, Caristi C, Gargiulli C, Bellocco E, Toscano G, et al. (2006) Flavonoid glycosides in bergamot juice (Citrus bergamia Risso). J Agric Food Chem 54: 3929-3935.

48. Shi P, He Q, Song Y, Qu H, Cheng Y (2007) Characterization and identification of isomeric flavonoid O-diglycosides from genus Citrus in negative electrospray ionization by ion trap mass spectrometry and time-of-flight mass spectrometry. Anal Chim Acta 598: 110-118.

49. Denekamp C, Claeys M, Pocsfalvi G (2000) Mechanism of cross-ring cleavage reactions in dirhamnosyl lipids: effect of the alkali ion. Rapid Commun Mass Spectrom 14: 794-799.

50. Cuyckens F, Rozenberg R, de Hoffmann E, Claeys M (2001) Structure characterization of flavonoid O-diglycosides by positive and negative nanoelectrospray ionization ion trap mass spectrometry. J Mass Spectrom 36: 1203-1210.

51. Zietz M, Weckmuller A, Schmidt S, Rohn S, Schreiner M, et al. (2010) Genotypic and climatic influence on the antioxidant activity of flavonoids in Kale (Brassica oleracea var. sabellica). J Agric Food Chem 58: 2123-2130.

52. Mabry TJ, Markham KR, Thomas MB (1970) The Systematic Identification of Flavonoids. 1st edn. New York: Springer-Verlag.

53. Ferreres F, Gil-Izquierdo A, Vinholes J, Silva ST, Valentao P, et al. (2012) Bauhinia forficata Link authenticity using flavonoids profile: relation with their biological properties. Food Chem 134: 894-904.

54. Vallejo F, Tomás-Barberán FA, Ferreres $F(2004)$ Characterisation of flavonols in broccoli (Brassica oleracea L. var. italica) by liquid chromatography-UV diode-array detection-electrospray ionisation mass spectrometry. J Chromatog A 1054: 181-193.

55. Ferreres F, Pereira DM, Valentao P, Andrade PB, Seabra RM, et al. (2008) New phenolic compounds and antioxidant potential of Catharanthus roseus. J Agric Food Chem 56: 9967-9974.

56. Motamedi H, Safary A, Maleki S, Seyyednejad SM (2009) Ziziphus spinachristi, a native plant from Khuzestan, Iran, as a potential source for discovery new antimicrobial agents. Asian J Plant Sci 8: 187-190.

57. Digrak M, Alma MH, Ilcim A, Sen S (1999) Antibacterial and antifungal effects of various commercial plant extracts. Pharm Biol 37: 216-220.

58. Huet O, Petit JM, Ratinaud MH, Julien R (1992) NADH-dependent dehydrogenase activity estimation by flow cytometric analysis of 3-(4,5-dimethylthiazolyl-2-yl)-2,5-diphenyltetrazolium bromide (MTT) reduction. Cytometry 13: 532-539.

59. Mosmann T (1983) Rapid colorimetric assay for cellular growth and survival: application to proliferation and cytotoxicity assays. J Immunol Methods 65: 55-63.

60. Fotakis G, Timbrell JA (2006) In vitro cytotoxicity assays: comparison of LDH neutral red, MTT and protein assay in hepatoma cell lines following exposure to cadmium chloride. Toxicol Lett 160: 171-177.

61. Weyermann J, Lochmann D, Zimmer A (2005) A practical note on the use of cytotoxicity assays. Int J Pharm 288: 369-376.

62. Uzunova VD, Cullinane C, Brix K, Nau WM, Day AI (2010) Toxicity of cucurbit[7] uril and cucurbit[8]uril: an exploratory in vitro and in vivo study. Org Biomol Chem 8: 2037-2042. 